\title{
Nonlinear optimal control for gust load alleviation with a physics-constrained data-driven internal model
}

\author{
Andrew Wynn *, Marc Artola ${ }^{\dagger}$, and Rafael Palacios * \\ Department of Aeronautics, Imperial College, London, UK, SW7 2AZ
}

\begin{abstract}
A data-driven strategy is developed to improve the internal models used for predictive control in nonlinear aeroelastic applications. A nonlinear modal formulation of the structure is retained, while an identified quadratic model for both the gravitational forces and the aerodynamics are obtained from a least-squares fit with LASSO regularisation from simulated flights. This is first seen to improve the accuracy of the resulting reduced-order model for openloop predictions on both gust response and a payload drop problem. Its superior performance as internal model for nonlinear control and estimation is finally demonstrated numerically.
\end{abstract}

\section{Introduction}

$\mathrm{T}$ HIS paper explores nonlinear control strategies for gust alleviation of aircraft with very flexible wings. The interest is on next-generation air vehicles driven by extreme efficiency considerations, such as very long endurance observation or communication platforms. The combination of very high wing aspect ratio to achieve aerodynamic efficiency and low loading to reduce structural weight, results in vehicles that may display complex dynamic response, including large wing deformations, in their interactions with a non-stationary atmosphere. For over two decades there has been a sustained effort to develop suitable modelling strategies to support their aeroelastic design [1], yet until recently most control strategies were still built on linear methods. Advanced control algorithms that exploit the nonlinear physics in the aircraft dynamics are instead desirable to enhance their manoeuvrability and/or reduce structural integrity risks (i.e., load alleviation).

Model predictive control (MPC) has been already shown [2-4] to be very suitable for this task, as it can optimally exploit arbitrary nonlinear dynamics and encode state and actuator constraints, while displaying provable stability guarantees. MPC relies on a suitable internal model of the relevant physics in the problem, upon which a constrained optimisation problem must be solved in real time (in fact, faster than real time) to define control actions using the available, and limited, measurements of the system state. The dual problem for MPC with limited sensor information is the Moving Horizon Estimator (MHE) problem, a second nonlinear programming problem that can be seen as a nonlinear extension of the Kalman filter. Both MPC and MHE are computationally intensive strategies, which benefit from both optimised algorithms [5] and problem formulations to deal with the relatively fast dynamics occurring in aircraft applications. Most MPC developments for nonlinear aeroelastic control have focused on defining suitable architectures using solely simulation. However, a first successful wind-tunnel demonstration of a gust load alleviation system using MPC with nonlinear constraints has been already carried by Barzgaran et al. [6].

A major challenge for the development of MPC/MHE for distributed parameter systems with complex nonlinear dynamics (with aeroelastic control of very flexible aircraft a prototypical example) is the efficient characterisation of the system dynamics with very few degrees of freedom. For applications to nonlinear control of very flexible structures, we have recently shown [7] that an intrinsic description of the geometrically-nonlinear composite beam equations produces a suitable formulation on which to build computationally-efficient MPC/MHE architectures. In particular, the linear normal modes of the structure provide an excellent basis for projection of the (geometrically nonlinear) intrinsic beam equations that results in a formulation with only quadratic nonlinearities. We have further developed that strategy for applications to nonlinear aeroservoelasticity, by augmenting the geometrically-nonlinear beam models with time-domain unsteady aerodynamics models linearised around an arbitrary equilibrium [8]. The starting point is the unsteady vortex-lattice method, possibly with steady corrections, which has been made tractable by the development of bespoke model-order reduction techniques [9, 10]. While that approach resulted in clear improvements in performance of the control system compared to those based on linear models, these results also made apparent that the linearised aerodynamics used in that approach restricted the general applicability of the approach to the challenging nonlinear

\footnotetext{
* Reader in Control and Optimisation, Room CAGB 340, South Kensington Campus (a.wynn@imperial.ac.uk)

†raduate research assistant, Room CAGB 308, South Kensington Campus (marc.artola16@imperial.ac.uk)

†Professor in Computational Aeroelasticity, Room CAGB 338, South Kensington Campus, Associate Fellow AIAA (r.palacios@imperial.ac.uk)
} 
aeroelastic control problem. Direct solution of the full nonlinear aeroelastic equations is, however, not possible within the internal model, and, consequently, the goal of the current paper is to investigate data-driven strategies to augment the aerodynamic fidelity of the internal models.

The paper continues with a quick summary of the aeroelastic modelling framework of [8] in section II] Section IIIIthen introduces our new data-driven approach to augment the fidelity of the nonlinear internal model. It considers first gravity forces, which are expensive to compute in the intrinsic beam model (the formulation is written in terms of strains, and rotations are computed only in postprocessing), before considering the aerodynamic loading resulting from large deformations. Finally, the approach is exemplified with a numerical study on a representative high-altitude long-endurance aircraft.

\section{Internal Aeroelastic Model for Control}

The following sections provide a brief overview of the aeroelastic modelling framework considered in this paper and will highlight components of the model for which data-driven modelling is later investigated.

\section{A. Intrinsic structural model}

We consider aircraft models whose structures can be described by jointed networks of composite beams. The dynamics of each beam in the network is described by the intrinsic formulation of geometrically exact beam theory [11]. In this formulation, the primary variables are the linear and angular inertial velocities $\boldsymbol{x}_{1}(s, t)=\left(\boldsymbol{v}^{\top}, \boldsymbol{\omega}^{\top}\right)^{\top}:[0, L] \times \mathbb{R}_{+} \rightarrow \mathbb{R}^{6}$ and the force and moment sectional resultants $\boldsymbol{x}_{2}(s, t)=\left(\boldsymbol{f}^{\top}, \boldsymbol{m}^{\top}\right)^{\top}:[0, L] \times \mathbb{R}_{+} \rightarrow \mathbb{R}^{6}$, which are defined in a local reference frame whose first unit vector $\boldsymbol{e}_{1}(s, t)$ is tangent to the (curved) reference line definining the beam geometry. Here, $L$ is the arclength of the undeformed beam, $s \in[0, L]$ the spatial coordinate parameterising its elastic axis and $t>0$ is time. The beam dynamics are governed by the nonlinear partial differential equations

$$
\begin{gathered}
M \frac{\partial \boldsymbol{x}_{1}}{\partial t}-\frac{\partial \boldsymbol{x}_{2}}{\partial s}-E x_{2}+\mathcal{L}_{1}\left(\boldsymbol{x}_{1}\right) M \boldsymbol{x}_{1}+\mathcal{L}_{2}\left(\boldsymbol{x}_{2}\right) C x_{2}=\boldsymbol{f}_{e} \\
C \frac{\partial \boldsymbol{x}_{2}}{\partial t}-\frac{\partial \boldsymbol{x}_{1}}{\partial s}+E^{\top} \boldsymbol{x}_{1}-\mathcal{L}_{1}\left(\boldsymbol{x}_{1}\right) C \boldsymbol{x}_{2}=0,
\end{gathered}
$$

where $M(s), C(s):[0, L] \rightarrow \mathbb{R}^{6 \times 6}$ are the symmetric positive definite mass and compliance matrices, $\boldsymbol{f}_{e}$ is an external forcing, and $E(s):[0, L] \rightarrow \mathbb{R}^{6 \times 6}$ includes the effect of initial curvatures and pre-twist $\kappa_{0}$ via

$$
E=\left(\begin{array}{cc}
\tilde{\boldsymbol{\kappa}}_{0} & 0 \\
\tilde{\boldsymbol{e}}_{1} & \tilde{\boldsymbol{\kappa}}_{0}
\end{array}\right),
$$

where $(\tilde{)})$ denotes the cross-product operator. Finally, $\mathcal{L}_{1}, \mathcal{L}_{2}: \mathbb{R}^{6} \rightarrow \mathbb{R}^{6 \times 6}$ are the linear operators

$$
\mathcal{L}_{1}\left(x_{1}\right)=\left(\begin{array}{cc}
\tilde{\omega} & 0 \\
\tilde{v} & \tilde{\omega}
\end{array}\right), \quad \mathcal{L}_{2}\left(x_{2}\right)=\left(\begin{array}{cc}
0 & \tilde{f} \\
\tilde{f} & \tilde{m}
\end{array}\right)
$$

and the PDE (1) is endowed with natural boundary conditions

$$
x_{1 i}(0, t) x_{2 i}(0, t)=0=x_{1 i}(L, t) x_{2 i}(L, t), \quad i=1, \ldots, 6 .
$$

Displacements $\boldsymbol{r}$ and rotations $T$ are secondary variables in the intrinsic formulation that may be required to describe certain external forcings, notably gravity. These are described with respect to a fixed inertial frame with $T(s, t):[0, L] \times \mathbb{R}_{+} \rightarrow \mathbb{R}^{3 \times 3}$ denoting the coordinate transformation matrix from the local to the inertial frame. It is convenient to express $T$ in terms of quarternions $\xi=\left[\xi_{v}^{\top}, \xi_{0}\right]^{\top} \in \mathbb{R}^{4}$ via

$$
T(\xi)=\left(\xi_{0}^{2}-\left\|\xi_{v}\right\|^{2}\right) I_{3}+2 \xi_{v} \xi_{v}^{\top}+2 \xi_{0} \tilde{\xi_{v}},
$$

with the relation to to the primary intrinsic variables given by

$$
\frac{\partial \xi}{\partial t}=\frac{1}{2}\left(\begin{array}{cc}
0 & -\omega^{\top} \\
\omega & -\tilde{\omega}
\end{array}\right) \xi=: \mathcal{U}(\omega) \xi, \quad \frac{\partial \boldsymbol{r}}{\partial t}=T \boldsymbol{v} .
$$


Model reduction of the structural equations is performed by taking a finite expansion of the primary variables $\boldsymbol{x}_{1}, \boldsymbol{x}_{2}, \zeta$ up to a truncation dimension $N$ by assuming that

$$
\boldsymbol{x}_{1}(s, t)=\sum_{i=1}^{N} \phi_{1 i}(s) q_{1 i}(t), \quad \boldsymbol{x}_{2}(s, t)=\sum_{i=1}^{N} \phi_{2 i}(s) q_{2 i}(t), \quad \boldsymbol{\xi}(s, t)=\sum_{i=1}^{N} \phi_{\xi i}(s) q_{\xi i}(t) .
$$

Typically, $\phi_{1 i}, \phi_{2 i}$ are selected from among the dominant vibration modes computed from the linearised, unforced, PDE (1), while $\phi_{\zeta i}$ are chosen from the eigenfunctions of the linearised equations of motion for the quarternions. We assume from here that such mode shapes are computed globally for the network of beams comprising a skeletal assembly for the modelled airframe. A standard Galerkin projection procedure of substituting the modal expansion into (1) then leads to an ODE of the following form describing the evolution of the modal coefficients $\left(\boldsymbol{q}_{1}, \boldsymbol{q}_{2}, \boldsymbol{q}_{\xi}\right) \in \mathbb{R}^{3 N}$ under gravity forcing [12]

$$
\begin{aligned}
\dot{\boldsymbol{q}}_{1} & =\boldsymbol{\Omega} \boldsymbol{q}_{2}-q_{1 \ell} \Gamma_{1}^{\ell} \boldsymbol{q}_{1}-q_{2 \ell} \Gamma_{2}^{\ell} \boldsymbol{q}_{2}+q_{\xi \ell} \Gamma_{g}^{\ell} \boldsymbol{q}_{\xi}+\boldsymbol{F}_{e}, \\
\dot{\boldsymbol{q}}_{2} & =-\boldsymbol{\Omega} \boldsymbol{q}_{1}+q_{2 \ell}\left(\Gamma_{2}^{\ell}\right)^{\top} \boldsymbol{q}_{1}, \\
\dot{\boldsymbol{q}}_{\xi} & =q_{1 \ell} \Gamma_{\xi}^{\ell} \boldsymbol{q}_{\xi},
\end{aligned}
$$

where Einstein notation has been used. Here, $\Omega \in \mathbb{R}^{N \times N}$ is a diagonal matrix containing the eigenvalues corresponding to the linear structural vibrations, $\Gamma_{1}, \Gamma_{2}, \Gamma_{\xi} \in \mathbb{R}^{N \times N}$ capture the quadratic nonlinearities of 11 and the quarternion dynamics, while $\Gamma_{g}$ encodes the gravity forcing. Finally, $\boldsymbol{F}_{e}$ is the modal projection of any remaining external forces (e.g. aerodynamics, actuation, gusts). We now describe the aerodynamic model to be coupled with (4).

\section{B. Aerodynamic Modelling}

For an aerodynamic model we employ the Unsteady Vortex Lattice Method (UVLM), which assumes an incompressible potential flow. In this method, a discrete lattice of vortex rings (each forming a panel) covers both the lifting surfaces and a thin wake of finite length shed from each lifting surface. The inputs to the model are the instantaneous shape of the lifting surfaces, which is given by the coordinates of the panel vertices, $\boldsymbol{\zeta}_{b}$. The underlying states are the circulation intensities $\boldsymbol{\Gamma}_{b}$ of the vortex rings associated with the lifting surface (bound panels), the circulation intensities $\boldsymbol{\Gamma}_{w}$ of wake vortex rings (wake panels), and, if a free-wake assumption is used, also the co-ordinate locations of the wake panels, $\zeta_{w}$.

UVLM solves for a non-penetration boundary condition of the induced velocity field at the centre of each bound panel, enforces the Kutta condition at the trailing edge of each lifting surface, and convects vorticity at the local induced flow velocity. The structure of the dynamics induced by these assumptions can be described in terms of an aerodynamic state $\boldsymbol{x}_{A}=\left(\boldsymbol{\Gamma}_{b}, \boldsymbol{\Gamma}_{w}, \dot{\boldsymbol{\Gamma}}_{b}, \boldsymbol{\zeta}_{w}\right)$ and by viewing the coordinates and velocities of the bound panels as inputs $\boldsymbol{u}_{A}=\left(\boldsymbol{\zeta}_{b}, \dot{\boldsymbol{\zeta}}_{b}\right)$, and the aerodynamic forces at the bound panel locations as outputs $\boldsymbol{y}_{A}$ [13]. The resulting equations of motion are typically expressed in discrete-time, are nonlinear and have the general input-output form

$$
f_{A}\left(\boldsymbol{x}_{A}^{n+1}, \boldsymbol{u}_{A}^{n+1}\right)=g_{A}\left(\boldsymbol{x}_{A}^{n}, \boldsymbol{u}_{A}^{n}\right), \quad \boldsymbol{y}_{A}=h_{A}\left(\boldsymbol{x}_{A}^{n}, \boldsymbol{u}_{A}^{n}\right) .
$$

Accurate UVLM models of this form typically require $O\left(10^{5}\right)$ states, which is a significant barrier for their use in control algorithms. Model reduction is therefore necessary. In first approximation, this can be achieved if the nonlinear UVLM equations are first linearised about a fixed point (a trim condition with frozen wake geometry) to obtain a system of the form

$$
\boldsymbol{x}_{A}^{n+1}=A \boldsymbol{x}_{A}^{n}+B \boldsymbol{u}_{A}^{n+1}, \quad \boldsymbol{y}_{A}=C \boldsymbol{x}_{A}^{n}+D \boldsymbol{u}_{A}^{n},
$$

where $A, B, C, D$ are matrices of appropriate dimensions and, with slight abuse of notation, we retain the state variable definitions to refer to perturbation variables from the chosen trim condition (noting that the assumption of a frozen wake geometry implies that it is no longer necessary for the wake coordinate $\zeta_{w}$ to appear in the aerodynamic state variable).

Model reduction can now be performed in two steps. The first makes use of modal projection to significantly reduce the dimension of the input and output spaces. In particular, for a fine discretisation of bound and wake panels, it is typical that $\boldsymbol{u}_{A}^{n} \in \mathbb{R}^{u}, \boldsymbol{y}_{A} \in \mathbb{R}^{p}$ with $u, p \gg 1$. Since $\boldsymbol{y}_{A}$ describes aerodynamic forcing at a grid of locations on each lifting surface, interpolation and projection onto the structural modes $\phi_{1 j}$ can be used to construct $K_{y} \in \mathbb{R}^{N \times p}$ such that an external forcing $\boldsymbol{\eta}_{a}=K_{y} \boldsymbol{y}_{A}$ captures the aerodynamic forcing in the modal ODE (4). Second, since the inputs to (6) are the (perturbed) coordinates and velocities of the bound panels, they can be expressed as a linear function 
of the (perturbed) structural intertial velocities $\boldsymbol{x}_{1}$, displacements $\boldsymbol{r}$, and quarternions $\boldsymbol{\xi}$. Model reduction is then possible by considering the modal expansion for $\boldsymbol{x}_{1}=\sum_{i} \phi_{1 i} \boldsymbol{q}_{1 i}$ and by considering a similar modal expansion of the displacements/rotations [7]

$$
\left(\begin{array}{l}
\boldsymbol{r} \\
\boldsymbol{\xi}
\end{array}\right)=\sum_{i=1}^{N} \phi_{0 i} q_{0 i} .
$$

Letting $\boldsymbol{q}_{0}$ be the vector of the displacement/rotation modal weights, it is then the case that there exists $K_{u} \in \mathbb{R}^{u \times 2 N}$ such that $\boldsymbol{u}_{A}=K_{u}\left(\boldsymbol{q}_{0}^{\top}, \boldsymbol{q}_{1}^{\top}\right)^{\top}$. The linearised aerodynamic system (6) can therefore be re-expressed as a high-dimensional system but with only $2 N$ inputs and $N$ outputs.

Model reduction of the aerodynamic state $\boldsymbol{x}_{A} \in \mathbb{R}^{n}$ can now be achieved by moment matching which seeks a low-order linear system whose transfer functions (and their moments) match at a given frequency [10]. By selecting this to be in the low-frequency range of importance to aircraft aerodynamics, one obtains a projection matrix $V \in \mathbb{R}^{N \times n}$ and a reduced-order aerodynamic state $\boldsymbol{q}_{\Gamma}:=V \boldsymbol{x}_{A} \in \mathbb{R}^{N}$. Upon projecting (6) and converting to continuous time, we obtain a reduced-order approximation to the UVLM aerodynamics

$$
\dot{\boldsymbol{q}}_{\Gamma}=A_{\Gamma} \boldsymbol{q}_{\Gamma}+B_{\Gamma}\left(\begin{array}{ll}
\boldsymbol{q}_{0}^{\top} & \boldsymbol{q}_{1}^{\top}
\end{array}\right)^{\top}, \quad \boldsymbol{\eta}_{a}=C_{\Gamma} \boldsymbol{q}_{\Gamma}+D_{\Gamma}\left(\begin{array}{ll}
\boldsymbol{q}_{0}^{\top} & \boldsymbol{q}_{1}^{\top}
\end{array}\right)^{\top}
$$

with reduced-order state-dimension $N$ and with $2 N$ inputs and $N$ outputs.

\section{Aeroelastic system}

Combining the aerodynamic reduced-order model (ROM) (7) with the structural ROM (4) and augmenting $D_{\Gamma}$ to include control inputs $\boldsymbol{u}$ (e.g. control surface deflections and propulsive thrusts) and external disturbances $\boldsymbol{w}$ (e.g. gusts) gives the combined aeroservoelastic ROM

$$
\begin{aligned}
\dot{\boldsymbol{q}}_{1} & =\Omega \boldsymbol{q}_{2}-q_{1 \ell} \Gamma_{1}^{\ell} \boldsymbol{q}_{1}-q_{2 \ell} \Gamma_{2}^{\ell} \boldsymbol{q}_{2}+\overbrace{q_{\xi} \Gamma_{g}^{\ell} \boldsymbol{q}_{\xi}}^{\text {gravity }}+\overbrace{C_{\Gamma} \boldsymbol{q}_{\Gamma}+D_{\Gamma}\left(\begin{array}{llll}
\boldsymbol{q}_{0}^{\top} & \boldsymbol{q}_{1}^{\top} & \boldsymbol{u}^{\top} & \left.\boldsymbol{w}^{\top}\right)^{\top}
\end{array},\right.}^{\text {aerodynamic forcing }}, \\
\dot{\boldsymbol{q}}_{2} & =-\Omega \boldsymbol{q}_{1}+q_{2 \ell}\left(\Gamma_{2}^{\ell}\right)^{\top} \boldsymbol{q}_{1}, \\
\dot{\boldsymbol{q}}_{0} & =\boldsymbol{q}_{1}, \\
\dot{\boldsymbol{q}}_{\xi} & =q_{1 \ell} \Gamma_{\xi}^{\ell} \boldsymbol{q}_{\xi}, \\
\dot{\boldsymbol{q}}_{\Gamma} & =A_{\Gamma} \boldsymbol{q}_{\Gamma}+B_{\Gamma}\left(\begin{array}{llll}
\boldsymbol{q}_{0}^{\top} & \boldsymbol{q}_{1}^{\top} & \boldsymbol{u}^{\top} & \left.\boldsymbol{w}^{\top}\right)^{\top},
\end{array}\right.
\end{aligned}
$$

which has approximately $5 N$ states. For the purposes of control implementation, a structural model with $N=O(10)$ states is sufficient to capture the dominant (and relevant) nonlinear structural dynamics. Such a model may be possible to use in a real-time implementable nonlinear model predictive control (NMPC) algorithm [8], but a number of deficiencies must first be addressed.

\section{Room for improvement in aeroelastic ROMs: a motivating example.}

With a view towards enabling real-time nonlinear control, two characteristics of the aeroservoelastic ROM (8) have potential to be improved. First, the requirement to model gravity and aerodynamics necessitates the use of additional state variables $\boldsymbol{q}_{\xi}, \boldsymbol{q}_{0}$ which increase the ROM dimension. If the dynamic influence of these variables can be approximated in terms of the primary variables $\boldsymbol{q}_{1}, \boldsymbol{q}_{2}, \boldsymbol{q}_{\Gamma}$ alone, further dimensional reduction can be achieved.

Second, model reduction of the aerodynamic subsystem (7) requires linearisation of the governing equations of UVLM. This implies that the aerodynamic model may only be accurate near to the chosen trim condition, in contrast to the underlying nonlinear structural model. To emphasise this point we consider a High-Altitude Long-Endurance concept, used by del Carre et al. and detailed in [14]. The HALE aircraft, shown schematically in Fig. 1(a), has a high aspect ratio (32) main wing and a T-tail mounted tailplane, where the control surfaces-a rudder and an all-moving elevator-are located. The weight of the aircraft is $78.25 \mathrm{~kg}$ including a $50 \mathrm{~kg}$ payload at the wing-fuselage intersection, which causes the aircraft wings to deflect significantly in trimmed flight. We now discuss the quality of a linear aerodynamic ROM designed for the full payload configuration, but after the payload is dropped from the aircraft. This 
test-case was discussed in detail in [8], where full details can be found, but, to motivate the new data-driven approach taken in this paper, we reproduce some relevant results here.

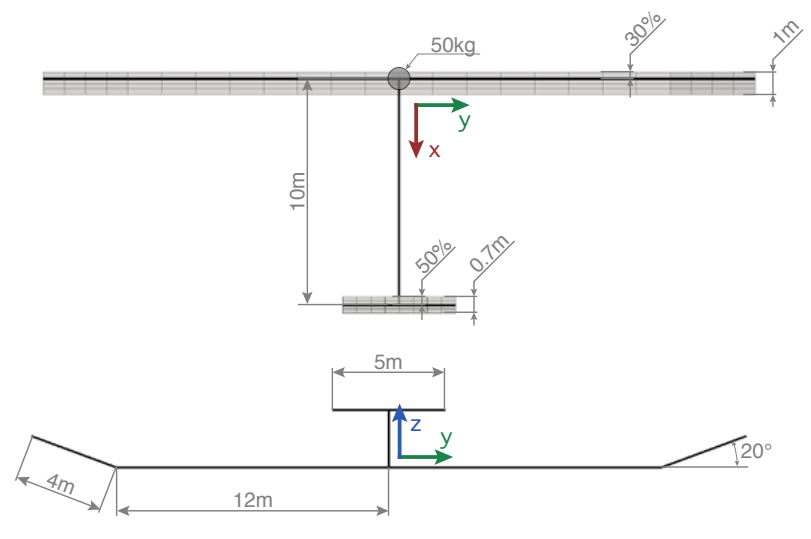

(a) 3-view schematic of HALE aircraft [14].

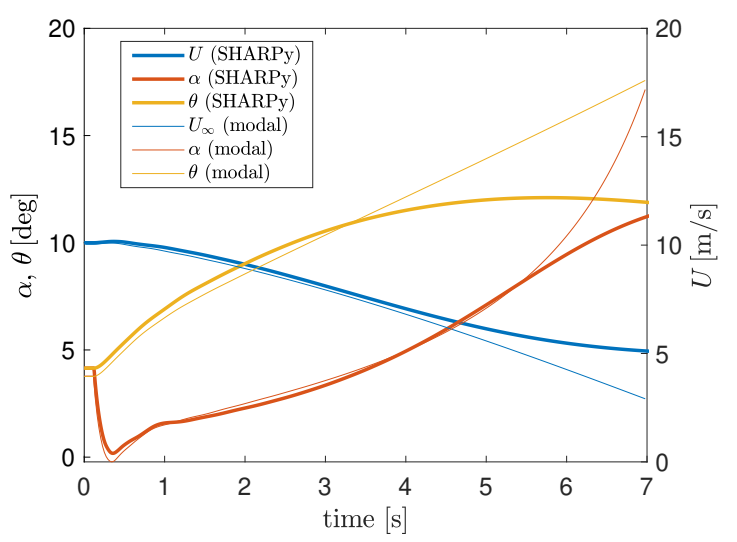

(b) Payload drop open-loop results

Fig. 1 HALE aircraft with change of linearisation.

Figure 1(b) shows predictions of the aircraft's instantaneous airspeed $U$ (measured as the velocity magnitude of a frame of reference, $A$, attached to the body at the location of the payload indicated in Figure 1(a)], angle of attack $\alpha$ (measured as the angle between the velocity of the frame $A$ and the horizontal axis of the same frame) and pitch angle $\theta$ (measured as the angle between the $x$-axis of the frame $A$ and the inertial horizontal direction). Simulations are performed using both a medium-fidelity aeroelastic model and a ROM of the form (8). The medium-fidelity model is simulatated using SHARPy [15] and employs a fully-nonlinear geometrically exact structural model coupled with a full-order nonlinear UVLM implementation, as described in Section II.C

The aircraft is initially trimmed with airspeed $U=10 \mathrm{~m} / \mathrm{s}$ but, after $0.125 \mathrm{~s}$, half of the $50 \mathrm{~kg}$ is dropped (instantaneously removed) which significantly alters the aircraft's dynamics. The sudden release of the payload causes an abrupt drop in angle of attack (due to the upwards velocity of the airframe as a reaction to the drop), which subsequently maintains a positive growth for the portrayed simulation window. The drastic change in the aircraft's centre of mass also leads to a progressive increase in pitch which, in the fully nonlinear SHARPy simulation (thick lines in Figure 1(b)), stabilizes after approximately five seconds. Finally, the velocity undergoes a substantial continuous decrease due to the climbing trajectory following the mass drop but, for the fully nonlinear aeroelastic simulation, also appears to stabilize towards the end of the displayed simulation window.

Results obtained using an aeroelastic ROM of the form (8), shown with thin lines in Figure 1(b), show very good agreement with the highly-resolved fully nonlinear simulations for up to 5 seconds. However, after this initial interval the results start to diverge. This discrepancy in airspeed arises since the linearised ULVM ROM (7) is transparent to variations in flight velocity (and hence dynamic pressure) and since linear approximations to rotations are employed, which are then subject to the follower-force effect when coupled with the nonlinear intrinsic structural equations. These features lead to an overestimate of drag forces due to an excessive horizontal projection of the lift during rigid body rotations, and the fact that the observed decrease in velocity after the mass drop does not translate into a reduction of the aerodynamic forces (through dynamic pressure).

Agreement for five seconds of open-loop simulation can be viewed positively and can successfully underpin efficient model predictive control (MPC) strategies [8]. However, it is certainly not desirable for predictive models to diverge within a relatively short simulation interval, especially if one would like to implement longer horizons in an MPC framework. For this reason, we now seek to identify nonlinear representations of both the "gravity", "aerodynamic forcing", and aerodynamic model components of (8) using data-driven modelling. We will seek to maintain the low model-order of the resulting ROM by only searching for quadratically-nonlinear representations of both processes. 


\section{A framework for data-driven aeroelastic modelling}

We now describe the general approach towards data-driven aeroelatic modelling taken in this paper, before detailing the data-acquisition process and introducing the specific test-cases, which are both considered in Section [V] Our aim, given ensembles of data corresponding to some or all states of the aeroelastic system $(8)$ is to identify the simplest possible nonlinear mappings that can replace both the gravity and aerodynamic forcing terms. This will be performed by searching for quadratically-nonlinear functions to replace both terms, implemented via a least-squares fit with $\ell_{1}$-penalty (LASSO regularization) to promote sparsity (i.e. simplicity) of the fitting polynomials. In both cased, fitting of quadratic polynomials is motivated by the underlying dynamics of both processes.

\section{A. Gravity modelling.}

The influence of gravity on 8 , given by $q_{\xi \ell} \Gamma_{g}^{\ell} \boldsymbol{q}_{\xi}=: \boldsymbol{\eta}_{g}$, requires knowledge of all quarternion modes, which implies that their temporal evolution must also be tracked in any accurate ROM. To attempt to reduce the dimensionality of this component of the model, note first that the instantaneous quarternion distribution $\boldsymbol{q}_{\xi}$ of the airframe can be computed by an appropriate spatial integration of $\boldsymbol{x}_{2}$, given knowledge of the rotational state at just a single point on the structure. It is therefore reasonable to expect that gravity forcing can be replaced by a function of $\boldsymbol{q}_{2}$ and a single tracked quarternion. If achieved, this reduces the dimension of the ROM by $N-4$ states (four states are still required to track the single quarternion) and expresses gravitational forcing more explictly in terms of the intrinsic structural variable $\boldsymbol{q}_{2}$. This may be of great advantage for analytical interrogation.

We now detail the data-driven approach to gravity modelling. First, pick an arbitrary point on the structure $s_{0}$ and track its associated quarternion $\boldsymbol{\xi}_{0}(t):=\boldsymbol{\xi}\left(s_{0}, t\right) \in \mathbb{R}^{4}$ evolution via

$$
\dot{\boldsymbol{\xi}}_{0}=q_{1 \ell} \Gamma_{0}^{\ell} \xi_{0}, \quad \Gamma_{0}^{\ell}:=\mathcal{U}\left(\phi_{1 \ell}\right) .
$$

We seek to construct a quadratic mapping $h_{g}: \mathbb{R}^{N+4} \rightarrow \mathbb{R}^{N}$ such that

$$
\boldsymbol{\eta}_{g}=q_{\xi \ell} \Gamma_{g}^{\ell} \boldsymbol{q}_{\xi} \approx h_{g}\left(\boldsymbol{\xi}_{0}, \boldsymbol{q}_{2}\right) .
$$

To do this using a data-driven approach, consider an ensemble $Y_{g}$ of the form

$$
Y_{g}:=\left\{\left(\left(\boldsymbol{\eta}_{g}\right)_{i},\left(\boldsymbol{\xi}_{0}\right)_{i},\left(\boldsymbol{q}_{2}\right)_{i}\right): 1 \leq i \leq M\right\}
$$

drawn from $M$ temporal snapshots of the full aeroelastic system, possibly sampled from multiple independent simulation runs-see Section IV.A for details. Next, given a vector $\boldsymbol{X} \in \mathbb{R}^{d}$, let $\Theta(\boldsymbol{X}) \in \mathbb{R}^{\frac{1}{2}(d+1)(d+2)}$ be a vector of all monomials formed by elements of $\boldsymbol{X}$ of quadratic or lower order. For example, if $d=2$ and $\boldsymbol{X}=\left\{z_{1}, z_{2}\right\}$ then

$$
\Theta(X)=\left(\begin{array}{llllll}
1 & z_{1} & z_{2} & z_{1}^{2} & z_{1} z_{2} & z_{2}^{2}
\end{array}\right)^{\top} \in \mathbb{R}^{6} .
$$

To identify $h_{g}$, we then simply pose a minimisation problem fitting quadratic combinations the $N+4$ components of the input variables $\left(\boldsymbol{\xi}_{0}, \boldsymbol{q}_{2}\right)$ to the observed gravity force $q_{\xi \ell} \Gamma_{g}^{\ell} \boldsymbol{q}_{\xi \ell}$. In particular, we penalise the least-squares fit, while promoting sparsity with an $\ell_{1}$ penalty:

$$
\min _{\boldsymbol{\beta}} \sum_{i=1}^{M}\left\|\left(\boldsymbol{\eta}_{g}\right)_{i}-\boldsymbol{\beta} \boldsymbol{\Theta}\left(\left(\boldsymbol{\xi}_{0}\right)_{i},\left(\boldsymbol{q}_{2}\right)_{i}\right)\right\|_{2}^{2}+\alpha\|\boldsymbol{\beta}\|_{1}, \quad \boldsymbol{\beta} \in \mathbb{R}^{N \times \frac{(N+5)(N+6)}{2}},
$$

where $\|\boldsymbol{\beta}\|_{1}:=\sum_{i, j}\left|\beta_{i j}\right|$. Letting $\boldsymbol{\beta}^{*}$ be the optimal decision variables, the data-driven approximation $h_{g}$ is given by

$$
h_{g}\left(\boldsymbol{\xi}_{0}, \boldsymbol{q}_{2}\right)=\boldsymbol{\beta}^{*} \boldsymbol{\Theta}\left(\boldsymbol{\xi}_{0}, \boldsymbol{q}_{2}\right) .
$$

The $\ell_{1}$-regularisation term $\alpha\|\beta\|_{1}$ with weight $\alpha>0$ can be tuned to trade-off sparsity promotion with the quality of the data-fit. 


\section{B. Aerodynamic force modelling}

Simplifying the aerodynamic subsystem presents a greater challenge than gravity modelling. Aerodynamic states $\boldsymbol{q}_{\Gamma}$ are both forced by, and themselves force, the structural modes via the last and first equations in (8), respectively, and models for both processes are required. To obtain them, suppose that a data ensemble of the form

$$
Y_{A}=\left\{\left(\left(\dot{\boldsymbol{q}}_{\Gamma}\right)_{i},\left(\boldsymbol{q}_{\Gamma}\right)_{i},\left(\boldsymbol{\eta}_{a}\right)_{i},\left(\boldsymbol{q}_{1}\right)_{i},\left(\boldsymbol{q}_{2}\right)_{i}, \boldsymbol{u}_{i}, \boldsymbol{w}_{i}\right): 1 \leq i \leq M\right\}
$$

has been collected, providing state information at $M$ timesteps drawn from one or more aeroelatic simulations. That is, the data ensemble for modelling includes values of the modal intrinsic states $\boldsymbol{q}_{1}, \boldsymbol{q}_{2}$, any control inputs $\boldsymbol{u}$ and external disturbances $\boldsymbol{w}$ and aerodynamic information $\left(\dot{\boldsymbol{q}}_{\Gamma}, \boldsymbol{q}_{\Gamma}, \boldsymbol{\eta}_{a}\right)$.

The exact form of the aerodynamic information requires some additional comment. We assume that all simulations used to create the data $Y_{A}$ employ a common aerodynamic model of arbitrary fidelity. One example, considered in this paper, is the UVLM model described in section II.C. Data $\left(\boldsymbol{\eta}_{a}\right)_{i}$ are then projections of the computed aerodynamic forces onto the structural modes $\phi_{1 j}, \phi_{2 j}$ corresponding to the amplitudes $\left(\boldsymbol{q}_{1}\right)_{i},\left(\boldsymbol{q}_{2}\right)_{i}$ through (3). Less obviously, it is assumed that the aerodynamic states $\boldsymbol{q}_{\Gamma}$ collected as part of the data ensemble are projections of the (typically) high-dimensional aerodynamic state onto a fixed subspace of dimension $N$. For example, the UVLM model described in section II.C is used, then the full order state $\boldsymbol{x}_{A} \in \mathbb{R}^{n}$ can be reduced by the projection matrix $V \in \mathbb{R}^{N \times n}$ where $n \gg N$. Importantly, we assume that the chosen projection matrix must be fixed across all simulations whose data is used to create the ensemble $Y_{A}$. Such a restriction is necessary for robust system identification, otherwise the underlying (reduced-order) aerodynamic states would not be comparable across the whole data ensemble.

This restriction poses a challenge. Common projection operators, such $V$ discussed above, typically arise from appealing to classical model reduction techniques applied to linearised aerodynamic models. In contrast we hope to create data-driven aerodynamic models which are accurate for a wide range of conditions. To obtain them, $Y_{A}$ must contain data exhibiting a wide range of aeroelastic dynamics, and should certainly not be restricted to data drawn from the neighbourhood of a single equilibrium position. For this reason employing a fixed projection (e.g. $V$ ) defined by appealing to linear theory may produce reduced-order aerodynamic states $\boldsymbol{q}_{\Gamma}$ whose ability to represent full-order aerodynamic features varies across the ensemble $Y_{A}$. We discuss later in Section IV.A.2 possible choices of projection operator which can help to mitigate this issue.

Data-driven aerodynamic modelling is implemented in an analogous manner to gravity modelling. Again, we seek quadratic models by solving

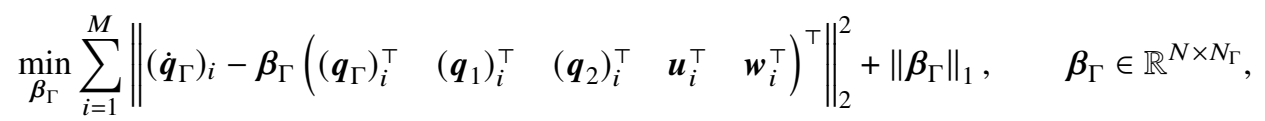

where $N_{\Gamma}=\frac{1}{2}\left(3 N+N_{u}+N_{w}+1\right)\left(3 N+N_{u}+N_{w}+2\right)$ and $N_{u}, N_{w}$ are the control input and external disturbance dimensions, respectively. Letting $\boldsymbol{\beta}_{\Gamma}^{*}$ be the optimal decision variable, we have the approximate aerodynamic model

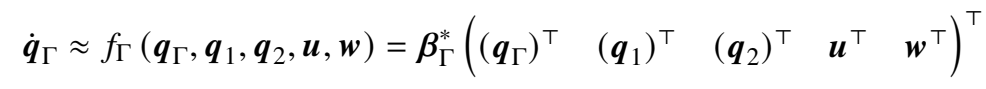

Finally, we also solve

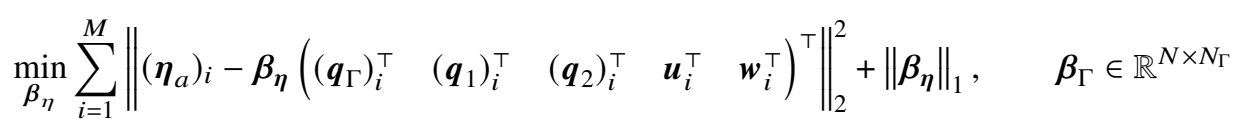

and let

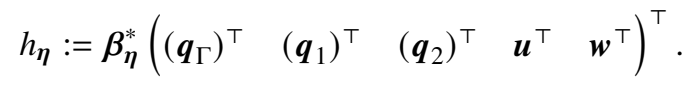

The data-driven approximating to the reduced-order aerodynamic model $(8)$ is then given by

$$
\begin{aligned}
& \dot{\boldsymbol{q}}_{1}=\Omega \boldsymbol{q}_{2}-q_{1 \ell} \Gamma_{1}^{\ell} \boldsymbol{q}_{1}-q_{2 \ell} \Gamma_{2}^{\ell} \boldsymbol{q}_{2}+h_{g}\left(\boldsymbol{\xi}_{0}, \boldsymbol{q}_{2}\right)+h_{\boldsymbol{\eta}}\left(\boldsymbol{q}_{\Gamma}, \boldsymbol{q}_{1}, \boldsymbol{q}_{2}, \boldsymbol{u}, \boldsymbol{w}\right), \\
& \dot{\boldsymbol{q}}_{2}=-\Omega \boldsymbol{q}_{1}+q_{2 \ell}\left(\Gamma_{2}^{\ell}\right)^{\top} \boldsymbol{q}_{1} \\
& \dot{\boldsymbol{\xi}}_{0}=q_{1 \ell} \Gamma_{0}^{\ell} \xi_{0}, \\
& \dot{\boldsymbol{q}}_{\Gamma}=f_{\Gamma}\left(\boldsymbol{q}_{\Gamma}, \boldsymbol{q}_{1}, \boldsymbol{q}_{2}, \boldsymbol{u}, \boldsymbol{w}\right),
\end{aligned}
$$


Note that this reduces the dimension of the aeroelastic ROM from $5 N$ states in $(8)$ to $3 N+4$ states of the system (12). However, the main potential advantage is that $f_{\Gamma}, h_{\eta}$ may allow identification of a nonlinear, and potentially sparse, representation of the aerodynamic subsystem. Furthermore, the ROM (12) is more compact than (8), in that it relies only upon a single "displacement/rotation" state $\boldsymbol{\xi}_{0}$. It is a modelling choice whether to include $\boldsymbol{\xi}_{0}$ in the Ansatz for the fitted functions $h_{\eta}$ and $f_{\Gamma}$ as a proxy for the state $\boldsymbol{q}_{0}$ in the original ROM. For dynamic simplicity, we do not choose to do so in this study, instead relying on the linear relation $\boldsymbol{q}_{0} \approx-\Omega^{-1} \boldsymbol{q}_{2}$ and the fitted nonlinearities contained in $f_{\Gamma}, h_{\eta}$ to capture the influence of $\boldsymbol{q}_{0}$ on the overall system dynamics.

\section{Data-driven modelling: HALE test-case}

We now implement the data-driven modelling framework described in Section III] Data-driven methods require specific data ensembles and these will be drawn from simulations of the HALE aircraft shown in Figure 1(a), and for which a full description can be found in [14]. The underlying structural modes $\phi_{1 i}, \phi_{2 i}$ which underpin the data-driven ROM are chosen as the first 17 normal modes of the aircraft, ordered by increasing natural frequency, from which 6 correspond to the different rigid body motions of the aircraft ( 3 translations plus 3 rotations about the aircraft principal axis of inertia). The remaining 11 elastic modes include 6 symmetric models (longitudinal dynamics) and 5 antisymmetric modes (lateral dynamics). These modes are computed for the full $50 \mathrm{~kg}$ payload configuration of the HALE aircraft.

Nonlinear aeroelastic simulations of the HALE aircraft, necessary to generate the high-dimensional aerodynamic data required for model fitting, are performed with the software package SHARPy using the parameters detailed in Ref. [8, Table 1]. We note only that a converged UVLM implementation for this test-case required 1792 bound panels, 17,920 wake panels, a time-step of $6.25 \mathrm{~ms}$. This SHARPy implementation is three orders of magnitude slower than the corresponding aeroelastic ROM (nonlinear structural/linearised aerodynamics) of the form (8), built using the structural modes described above and following the procedure of Section [I] [8]. Consequently, if fully nonlinear aeroelastic ROMs of the form (12) can be identified at similar, or lower, model dimension then this would provide significant computational advantages for the prediction and control of aeroelastic systems with geometrically nonlinear structural deformations.

\section{A. Data acquisition}

The necessary data ensembles $Y_{A}$ for model training will be created by first prescribing an ensemble of structural motions and deformations (i.e. in both the rigid-body and elastic modes of the aircraft) and, for each case, simulating the response of a resolved UVLM wake to this prescribed structural motion. Successful data-driven model identification requires a rich training ensemble that exhibits aerodynamic behaviour from a range of possible motions and deformations of the aircraft.

To attempt to achieve this, we assume in this paper that structural trajectories are chosen to be sinusoidal perturbations to a typical trim condition of the HALE aircraft. This is detailed in Section IV.A.2 It is desirable to prescribe these perturbations in terms of the underlying modes of the structure, since this will give an efficient and systematic method of defining physically reasonable structural deformations. A minor technical issue that must first be overcome is how to translate perturbations defined in modal dynamics into perturbations that can be implemented in a higher-fidelity aeroelastic solver.

\section{Obtaining high-fidelity aerodynamic data corresponding to prescribed structural modal inputs.}

The first step is to translate intrinsic modal coordinates $\boldsymbol{x}_{1}=\left(\boldsymbol{v}^{\top}, \omega^{\top}\right)^{\top}, \boldsymbol{x}_{2}=\left(\boldsymbol{f}^{\top}, \boldsymbol{m}^{\top}\right)^{\top}$ into the primary variables of the higher-fidelity aeroelastic solver SHARPy. SHARPy couples an FE-based implementation of the geometrically exact structural PDE (1), but using displacements and rotations as primary variables, with UVLM aerodynamics. In particular, elastic displacements $\boldsymbol{R}_{A}$ and rotations $\boldsymbol{\Psi}_{A}$ are used as primary structural variables, given with respect to the body-attached frame of reference $A$, whose linear and angular velocities $v_{A}, \omega_{A}$ and orientation with respect to an inertial frame of reference are also required. The relationship between this set of variables and the intrinsic local velocities $\boldsymbol{x}_{1}=\left(\boldsymbol{v}^{\top}, \omega^{\top}\right)^{\top}$ is given by

$$
\begin{aligned}
\boldsymbol{v}(s, t) & =T\left(\boldsymbol{\Psi}_{A}(s, t)\right) \dot{\boldsymbol{R}}_{A}(s, t)+T\left(\boldsymbol{\Psi}_{A}(s, t)\right)\left(\boldsymbol{v}_{A}(t)+\tilde{\boldsymbol{\omega}}_{A}(t) \boldsymbol{R}_{A}(s, t)\right), \\
\boldsymbol{\omega}(s, t) & =T_{t}\left(\boldsymbol{\Psi}_{A}(s, t)\right) \dot{\boldsymbol{\psi}}_{A}(s, t)+T_{t}\left(\boldsymbol{\Psi}_{A}(s, t)\right) \boldsymbol{\omega}_{A}(t),
\end{aligned}
$$


while the local strains and curvatures $\left(\boldsymbol{\gamma}^{\top}, \boldsymbol{\kappa}^{\top}\right)^{\top}=C \boldsymbol{x}_{2}$ are related via the following

$$
\begin{aligned}
& \boldsymbol{\gamma}(s, t)=T\left(\boldsymbol{\Psi}_{A}(s, t)\right) \boldsymbol{R}_{A}^{\prime}(s, t)+T\left(\boldsymbol{\Psi}_{A}(s, 0)\right) \boldsymbol{R}_{A}^{\prime}(s, 0), \\
& \boldsymbol{\kappa}(s, t)=T_{t}\left(\boldsymbol{\Psi}_{A}(s, t)\right) \boldsymbol{\Psi}_{A}^{\prime}(s, t)+T_{t}\left(\boldsymbol{\Psi}_{A}(s, t)\right) \boldsymbol{\Psi}_{A}^{\prime}(s, 0) .
\end{aligned}
$$

Here, $T\left(\boldsymbol{\Psi}_{A}(s, t)\right)$ is the transformation matrix between the local frame of reference and the body-attached frame $A$ [16] and $T_{t}\left(\Psi_{A}(s, t)\right)$ is the tangential rotation operator [17].

In Section IV.A.2, we will create training data ensembles of aerodynamic data corresponding to structural perturbations from a given aircraft trim condition. Consequently, we begin by using SHARPy to maintain trimmed flight of the aircraft (at, for example, a given airspeed) and extract the corresponding trim variables $\overline{\boldsymbol{R}}_{A}, \overline{\boldsymbol{\Psi}}_{A}, \overline{\boldsymbol{v}}_{A}$ and $\overline{\boldsymbol{\omega}}_{A}$. In addition, nodal aerodynamic forces $\boldsymbol{y}_{A}$ and gravity forces $\boldsymbol{y}_{g}$ corresponding to trim are found at the UVLM nodes. Using the intrinsic mode shapes $\phi_{1 i}, \phi_{2 i}$, these forces can then be converted into modal force inputs $\overline{\boldsymbol{\eta}}_{A}, \overline{\boldsymbol{\eta}}_{g}$ and, subsequently, the modal trim values $\overline{\boldsymbol{q}}_{1}, \overline{\boldsymbol{q}}_{2}$ are found by finding the steady solution to (4) but with $\boldsymbol{F}_{e}$ replaced by $\overline{\boldsymbol{\eta}}_{A}$ and gravity forces replaced by $\overline{\boldsymbol{\eta}}_{g}$.

Physically reasonable perturbations to the modal trim solutions can then be prescribed in the form $\boldsymbol{q}_{1}(t)=$ $\overline{\boldsymbol{q}}_{1}+\tilde{\boldsymbol{q}}_{1}(t), \boldsymbol{q}_{1}(t)=\overline{\boldsymbol{q}}_{2}+\tilde{\boldsymbol{q}}_{2}(t)$, which will be detailed in Section IV.A.2 After converting these inputs to the variables $(\boldsymbol{v}, \omega, \gamma, \boldsymbol{\kappa})$ using the intrinsic mode shapes, spatial integration of (14) gives the displacement and rotation fields $\boldsymbol{R}_{A}, \boldsymbol{\Psi}_{A}$ corresponding to the prescribed perturbations. The time-derivative counterparts $\dot{\boldsymbol{R}}_{A}, \dot{\boldsymbol{\Psi}}_{A}$ are easily obtained from (13), once the rigid-body velocities of the frame $A$ are computed from

$$
\left(\begin{array}{c}
\boldsymbol{v}_{A}(t) \\
\omega_{A}(t)
\end{array}\right)=\sum_{j=1}^{N} \phi_{1 j}\left(s_{A}\right) q_{1 j}(t),
$$

where $s_{A}$ is the location $s_{A}$ of the body-attached frame of reference. Adding control surface deflection inputs to the obtained $\boldsymbol{R}_{A}(t), \boldsymbol{\Psi}_{A}(t)$ and $\dot{\boldsymbol{R}}_{A}(t), \dot{\boldsymbol{\Psi}}_{A}(t), \boldsymbol{v}_{A}, \boldsymbol{\omega}_{A}$ all the necessary data for a fully prescribed UVLM simulation is ready, where only the UVLM module is run in SHARPy for the obtained structural time-histories. Finally, a small correction to account for the error introduced by expansions $(3)$ is added to the displacement and rotation time-histories before they are fed as an input to the simulations in SHARPy. These correction terms, namely $\overline{\boldsymbol{R}}_{A}-\boldsymbol{R}_{A}(0), \overline{\mathbf{\Psi}}_{A}-\boldsymbol{\Psi}_{A}(0),-\dot{\boldsymbol{R}}_{A}(0),-\dot{\boldsymbol{\Psi}}_{A}(0)$ and those for the velocities of the frame $A$, namely, $\overline{\boldsymbol{v}}_{A}-\boldsymbol{v}_{A}(0)$ and $-\omega_{A}(0)$ ensure that simulations start from SHARPy's original trim conditions, effectively removing any accumulated errors after the described manipulation.

The outcome of SHARPy's UVLM simulations are time histories of aerodynamic forces $\boldsymbol{f}_{a}(t)$ and gravitational forces which, upon projection, give time series of modal aerodynamic forces $\boldsymbol{\eta}_{a}(t) \subset \mathbb{R}^{N}$ and gravity forces $\boldsymbol{\eta}_{g}(t) \subset \mathbb{R}^{N}$. In addition, full-order aerodynamic state trajectories $\boldsymbol{x}_{A}(t)$ and their time derivatives $\dot{\boldsymbol{x}}_{A}(t)$ are obtained (c.f. Section II.C).

\section{Training data definition: HALE test-case}

Data ensembles are created from 94 simulations of $10 \mathrm{~s}$ each. Each simulation run corresponds to a perturbed trajectory from a nonlinear trim condition given by $(\bar{\alpha}, \bar{U}, \bar{\theta})=(4.1566 \mathrm{deg}, 10 \mathrm{~m} / \mathrm{s}, 4.1566 \mathrm{deg})$ in the angle of attack, airspeed and pitch angle. To ensure that the data ensemble is physically realistic, perturbations will be restricted to the ranges $\pm 10 \mathrm{deg}, \pm 6 \mathrm{~m} / \mathrm{s}$ and $\pm 20 \mathrm{deg}$, in $\alpha, U$ and $\theta$, respectively. Perturbation time-histories $\alpha(t), U(t), \theta(t)$ are generated using $1-\cos$ perturbations to the trim values. Each of the amplitudes for the $1-\cos$ perturbations are randomly chosen, using a uniform probability distribution within the defined intervals. For the frequencies, random values using the same probability distribution are chosen from the interval $[0.067,0.2] \mathrm{Hz}$. This is in line with the observed slow flight dynamics of the vehicle. The angle of attack, flight velocity and pitch amplitudes and frequencies are chosen for each variable independently. The resulting time histories can be related to the velocity of the body-attached frame of reference A, which can then be used to define a time history for the first six (rigid-body) modes $\boldsymbol{q}_{1}^{\text {rb }}$ of the $\boldsymbol{q}_{1}$ vector,

$$
\boldsymbol{q}_{1}^{\mathrm{rb}}=\boldsymbol{\Phi}_{1 v}^{-1}\left(\begin{array}{c}
-U(t) \cos (\alpha(t)) \\
0 \\
-U(t) \sin (\alpha(t)) \\
0 \\
\dot{\theta}(t) \\
0
\end{array}\right) .
$$


The remaining modal inputs (i.e., symmetric and longitudinal elastic modal velocities and elastic forces/moments) are also prescribed using perturbations with amplitudes $\pm(5,3,3,3,2,1)^{\top}$ and $\pm(20,8,3,2,2,1)^{\top}$, respectively, with 1 - cos signals of slightly faster random frequencies in the range $[0.13,0.4] \mathrm{Hz}$ to the modal trim solutions $\boldsymbol{q}_{1}$ and $\boldsymbol{q}_{2}$. Similar to the construction of the flight mechanics (rigid-body modes) perturbations, all elastic amplitudes and frequencies are (randomly) chosen for each mode independently, therefore for each excitation time-history all amplitudes and frequencies are distinct and uncorrelated between modes. The amplitudes for these perturbations are selected to cover the range of observed typical responses in representative application problems where the model is to be employed. Here, since the internal models will be tested against gust perturbations, a range of open-loop simulations with gusts of different intensities and spans have been post-processed (i.e., the obtained structural data has been projected onto modal coordinates, after transformation to the intrinsic variables) to quantify the expected excitation ranges. Perturbations on elevator input are also defined with random amplitudes between $\pm 7.5 \mathrm{deg}$ around the trim value and frequencies in the range $[0.13,0.4] \mathrm{Hz}$ for the $1-$ cos temporal signals.

The time-histories of modal inputs, $\boldsymbol{q}_{1}(t), \boldsymbol{q}_{2}(t)$, which excite all modes simultaneously, are then transformed to nodal displacements, $\boldsymbol{R}_{A}$, and finite rotations, $\boldsymbol{\Psi}_{A}$, as described in Section IV.A.1 These are used to solve for the fully nonlinear UVLM equations with prescribed structural motions in SHARPY. Each of the simulations provides us with the aerodynamic solution, consisting of the state variable $\boldsymbol{x}_{A}=\left(\boldsymbol{\Gamma}_{b}, \boldsymbol{\Gamma}_{w}, \dot{\boldsymbol{\Gamma}}_{b}, \boldsymbol{\xi}_{w}\right)$ as discussed in Section II.C. In addition to the full aerodynamic state, the SHARPY simulations driven by prescribed structural motions also yield the distribution of aerodynamic and gravitational forces (see Section IV.A.1), which after projection onto modal coordinates are used to obtain a data-driven approximation for the modal aerodynamic and gravitational forces.

As discussed in Section III.B. several choices can now be made regarding the projection matrix used to reduce the high-dimensional aerodynamic state space on to smaller subspaces, in order to obtain concise UVLM descriptions via data-driven model regression. One option could be to use the projection matrices obtained from a model-order reduction (e.g., either Krylov-based or frequency-limited balanced methods) of the linearised UVLM equations. Then, as described in II.C. reduced-order states $\boldsymbol{q}_{\Gamma}$ associated with the UVLM data can be constructed using the projection matrix $V$. This method is not followed in this paper, however, since the perturbations from the linearisation point (trim condition) of the data ensemble are very large, and hence this projection may be far from optimal. Moreover, circulation derivatives are found to be very noisy in the computed nonlinear simulations, introducing an undesirable level of distortion to the training data.

Instead, we now take a more traditional data-driven approach. Taking inspiration from the fully nonlinear state-space representation of the UVLM equations (5), where the derivatives of the bound circulations are used to augment the state only because the forces (output) depend on them (they are not needed for the time-marching of the pure UVLM equations), we perform a singular value decomposition of the UVLM state ensembles (i.e. a concatenated matrix $Y_{\text {ae }}$ whose columns include all $M$ timesteps of available data from across the 94 simulations) excluding the circulation time-derivatives

$$
U \Sigma W^{\top}=Y_{\mathrm{ae}}=\left(\left(\begin{array}{l}
\boldsymbol{\Gamma}_{b} \\
\boldsymbol{\Gamma}_{w} \\
\xi_{w}
\end{array}\right)_{1}-\left(\begin{array}{l}
\overline{\boldsymbol{\Gamma}}_{b} \\
\overline{\boldsymbol{\Gamma}}_{w} \\
\overline{\boldsymbol{\xi}}_{w}
\end{array}\right), \quad \cdots \quad, \quad\left(\begin{array}{l}
\boldsymbol{\Gamma}_{b} \\
\boldsymbol{\Gamma}_{w} \\
\boldsymbol{\xi}_{w}
\end{array}\right)_{M}-\left(\begin{array}{l}
\overline{\boldsymbol{\Gamma}}_{b} \\
\overline{\boldsymbol{\Gamma}}_{w} \\
\overline{\boldsymbol{\xi}}_{w}
\end{array}\right)\right),
$$

where the trim variable values have been subtracted. Remarkably, given the typically high dimension (for the HALE aircraft, the UVLM state dimension is 78,015 ) of the underlying UVLM data, only a very small number of singular vectors (the columns of $U$ ) are needed to describe a significant proportion of the aerodynamic data. Indeed, for the specific data ensemble considered here, the first 10 singular values (the diagonal entries of $\Sigma$ ) satisfy

$$
\frac{\sum_{i=1}^{10} \sigma_{i}^{2}}{\sum_{i=1}^{M} \sigma_{i}^{2}}>0.995
$$

indicating that $99.5 \%$ of variance of the aerodynamic states can be described with only 10 degrees of freedom. Therefore, we let $V_{P} \in \mathbb{R}^{78015 \times 10}$ be the projection operator whose columns are the first 10 orthogonal columns of $U$ (also commonly referred to as the POD (Proper Orthogonal Decomposition) modes of the data ensemble). The corresponding reduced-order aerodynamic states, at each of the sample points $i$, are then defined by

$$
\left(\left(\boldsymbol{q}_{\Gamma}\right)_{1} \quad, \quad \cdots \quad, \quad\left(\boldsymbol{q}_{\Gamma}\right)_{M}\right)=V_{P}^{\top} Y_{\mathrm{ae}},
$$

and the associated time derivative data $\left(\dot{\boldsymbol{q}}_{\Gamma}\right)_{i}$ can be obtained similarly. 
Now that SVD-based aerodynamic states have been defined, all information for the data-ensembles $Y_{g}$ and $Y_{A}$ required for data-driven modelling framework of Section [II] is available. In particular, we let

$$
Y_{A}=\left\{\left(\left(\dot{\boldsymbol{q}}_{\Gamma}\right)_{i},\left(\boldsymbol{q}_{\Gamma}\right)_{i},\left(\boldsymbol{\eta}_{a}\right)_{i},\left(\boldsymbol{q}_{1}\right)_{i},\left(\boldsymbol{q}_{2}\right)_{i},\left(\delta_{e}\right)_{i},\left(\dot{\delta}_{e}\right)_{i}\right): 1 \leq i \leq M\right\}
$$

where $\delta_{e}, \dot{\delta}_{e}$ are the elevator deflections and deflection rates used to perturb the trimmed flight condition, at the corresponding sampling points. In addition, we let

$$
Y_{g}=\left\{\left(\left(\boldsymbol{\eta}_{g}\right)_{i},\left(\boldsymbol{\xi}_{0}\right)_{i},\left(\boldsymbol{q}_{2}\right)_{i}\right): 1 \leq i \leq M\right\},
$$

where the single tracked quarternion $\xi_{0}(\cdot)=\boldsymbol{\xi}\left(s_{A}, \cdot\right)$ used for model-fitting is chosen to have location $s_{A}$, i.e., at the position of the body-attached frame (payload location) indicated in Figure 1(a)

\section{B. Data-driven model identification}

We implement the optimisation framework described in Section [II] on the data ensembles constructed in Section IV.A.1 A pre-processing step is performed to normalise each variable so that each lies in the interval $[-1,1]$. This is achieved for each particular element $x_{i}$ of an ensemble $\left\{x_{i}\right\}_{i=1}^{M}$ by letting

$$
\check{x}_{i}:=-1+2 \frac{x_{i}-\min _{j}\left\{x_{j}\right\}_{j=1}^{M}}{\max _{j}\left\{x_{j}\right\}_{j=1}^{M}-\min \left\{x_{j}\right\}_{j=1}^{M}}, \quad i=1, \ldots, M,
$$

where $(\because)$ denotes a normalised value. Normalised versions of the three optimisation problems (9), (10) and (11) are solved. In addition, we impose further constraints on each optimisation problems to attempt, as far as is practiable, to encode physically meaningful behaviour into the fitted models. First, we constrain the trim inputs to remain a fixed point of our data-driven model, which can be expressed as a linear combination of the model coefficients. Second, we constrain the quadratic terms associated to the reduced aerodynamic states to be energy-preserving [18, Eq. 2.4], taking as the energy the product $\boldsymbol{q}_{\Gamma}^{\top} \boldsymbol{q}_{\Gamma}$, and we force all the nonlinear couplings between $\boldsymbol{q}_{\Gamma}$ and the rest of the inputs to be zero. This allows us to check for global boundedness of the obtained data-driven models following the approach of [18]. There, sufficient conditions to prove global boundedness of ordinary differential equations with quadratic nonlinear terms are given. These conditions, gathered in [18, Theorem 1], require negative definiteness of the linear terms of a shifted system (which involves linear and quadratic terms of the original system), which can be easily checked a posteriori by solving an LMI-constructed with the matrices obtained from the data-driven coefficients. If these condition are satisfied, it follows that the system exhibits a trapping region to which all solutions are globally attractive, ensuring global stability of the underlying system.

While it is unclear whether our low-order models of the UVLM equations should necessarily satisfy the imposed particular structure (energy-preserving nonlinear terms and uncoupled quadratic terms involving $\boldsymbol{q}_{\Gamma}$ and the other non-autonomous inputs), this allows us to guarantee that the data-driven models are stable for fixed inputs $\left(\boldsymbol{q}_{1}, \boldsymbol{q}_{2}, \delta_{e}, \dot{\delta}_{e}\right)$, which is critical for MPC internal simulations, without excessive penalisation on the model fitting error. Nevertheless, this stability definition is not very restrictive (it encompasses systems with globally stable limit cycles or fixed points, for instance) and following physical intuition it enables us to guarantee stability of the data driven reduced-order models. For the aircraft examples considered in this paper, it is observed that the the fully-nonlinear UVLM equations appear to be globally stable, in the sense that for a prescribed arbitrary structural configuration (i.e., for fixed inputs to the UVLM) the solution always shows convergence (i.e., circulation intensities converge to steady values and the wake panels stabilise in a particular, fixed layout). It is then reasonable to expect that the reduced-order models should inherit similar stability characteristics, although it is important to note that without imposing constraints (as discussed above) data-driven methods provide no guarantees of stability even in this setting.

The results of the fitting optimisation, implemented using Matlab's fitrlinear function, are gathered in table 1 , where the maximum error values shown are over the different output equations and all magnitudes refer to the normalised regression problem and coefficients, except for the second-to-last row, which shows the actual mean squared error. This is computed as the squared error averaged over the ensemble size.

The regression problems show, in general, very low normalised errors, which is an indication that the quadratic polynomial expressions offer good data-driven approximation to the input-output mappings that we seek. As said 


\begin{tabular}{lccc} 
& Gravity force $h_{g}$ & Aerodynamic ROM $f_{\Gamma}$ & Aerodynamic output $h_{\boldsymbol{\eta}}$ \\
\hline Lasso penalty $\alpha$ & $10^{-6}$ & $10^{-6}$ & $10^{-6}$ \\
Max. sparsity & $3.51 \%$ & $2.96 \%$ & $45.81 \%$ \\
Coefficients tolerance & $10^{-8}$ & $10^{-12}$ & $10^{-9}$ \\
Gradient tolerance & $10^{-8}$ & $10^{-12}$ & $10^{-9}$ \\
Number of coefficients & 57 & 7570 & 406 \\
Number of constraints & 0 & 5440 & 0 \\
Max. norm error & $3.6 \cdot 10^{-5}$ & $7.03 \cdot 10^{-3}$ & $1.19 \cdot 10^{-4}$ \\
Max. error & $4.49 \cdot 10^{-3}$ & 518.00 & 4.07 \\
Max. no. iterations & 85 & 7495 & 50,000 (reached iteration limit) \\
\hline
\end{tabular}

Table 1 Data-driven modelling: optimisation parameters and outputs.

before, constraining the regression problem for the dynamics of the low-order aerodynamic states (Table 1 . first column) does not significantly hinder the quality of the fit; the maximum normalised error of the unconstrained regressions is already $4.3 \cdot 10^{-3}$, that is, $39 \%$ lower that of the constrained one, however both are of the same order of magnitude. An interesting observation is that in the aerodynamic force output equation (Table 1 . final column), the achieved sparsity in the model coefficients is much higher than in the rest of the regressions, possibly highlighting the identification of patterns in the data such as aerodynamic stiffness and damping, which directly link the forces to the modal coefficients $\boldsymbol{q}_{2}$ and $\boldsymbol{q}_{1}$, respectively. Finally, the unsteady aerodynamics time-evolution model (Table 11. middle column) has been shown to exhibit a trapping region by virtue of the conditions in [18], which ensure global stability of the system for fixed inputs $\left(\boldsymbol{q}_{1}, \boldsymbol{q}_{2}, \delta_{e}, \dot{\delta}_{e}\right)$.

\section{Open-Loop Improvements}

The obtained data-driven models for the gravity forcing and for the low-order nonlinear UVLM are fitted in our aeroelastic internal model (12). The equation for the quaternion field approximation $\dot{\boldsymbol{q}}_{\xi}=q_{\xi}^{l} \Gamma_{\xi}^{l} \boldsymbol{q}_{1}$ is substituted by the evolution equation of a single quaternion $\xi_{0}$, for which we have chosen the quaternion associated to the rotation of the body-attached frame $A$, and the modal gravity forcing term $q_{\xi}^{l} \Gamma_{g}^{l} \boldsymbol{q}_{\boldsymbol{\xi}}$ is replaced by $h_{g}\left(\boldsymbol{\xi}_{0}, \boldsymbol{q}_{2}\right)$. Regarding the UVLM model, the linear matrices $\left(A_{\Gamma}, B_{\Gamma}, C_{\Gamma}, D_{\Gamma}\right)$ in $(8)$ are substituted by the data-driven expressions $f_{\Gamma}$ and $h_{\boldsymbol{\eta}}$.

Next, we compare the response of the fully-coupled internal aeroelastic system employing the described data-driven sub-components to ground truth fully nonlinear aeroelastic simulations obtained with SHARPY and also to our original internal model based on linearised UVLM low-order models (8). The data-driven model for gravity introduces no perceptible changes in the overall dynamics when compared to the modal-based quaternion and gravity approximations. Its main contribution, hence, is the simplification of the model of gravity within the modal intrinsic formulation. Therefore, the comparison below aims to highlight, qualitatively, the potential of the data-driven model of the unsteady aerodynamics to improve model accuracy.

For this comparison exercise, two test cases are considered, with large rigid-body motions which should reveal the limitations of the original, linear-aerodynamics-based internal models and the improvements of the data-driven additions. The first is the payload drop case discussed in Section II.D, which has already highlighted the model's shortcomings. The second, is chosen to be the response of the aircraft to an increment of elevator deflection of $-1 \mathrm{deg}$ for $4 \mathrm{~s}$ from trim conditions. This causes the aircraft to exhibit nose-up pitch behaviour, reducing its flight velocity and increasing the angle of attack. Such behaviour is known to cause divergent results using our original internal models.

The comparisons are gathered in Fig. 2. The original internal model, based on a linear UVLM ROM (thin solid line in Fig. 2), diverges dramatically in the payload drop case (top figure), with eventual blowup of the solution after the first few seconds of simulation. We see, that for this case, the model fitted with the data-driven gravity and aerodynamic terms (dashed lines) substantially improves the initial response and most importantly, the solution remains bounded showing very reasonable agreement with the full nonlinear simulations. Regarding the elevator input case (bottom figure), the original model again shows similar behaviour, however this time producing a bounded solution. This is characterised by a response with an apparent overestimation of drag, where the flight velocity and the angle of attack experience an exaggerated response; the former is reduced to values of around $6 \mathrm{~m} / \mathrm{s}$ and the latter almost doubles the maximum observed angle of attack of the ground truth simulation. We see that the data-driven enhanced model 

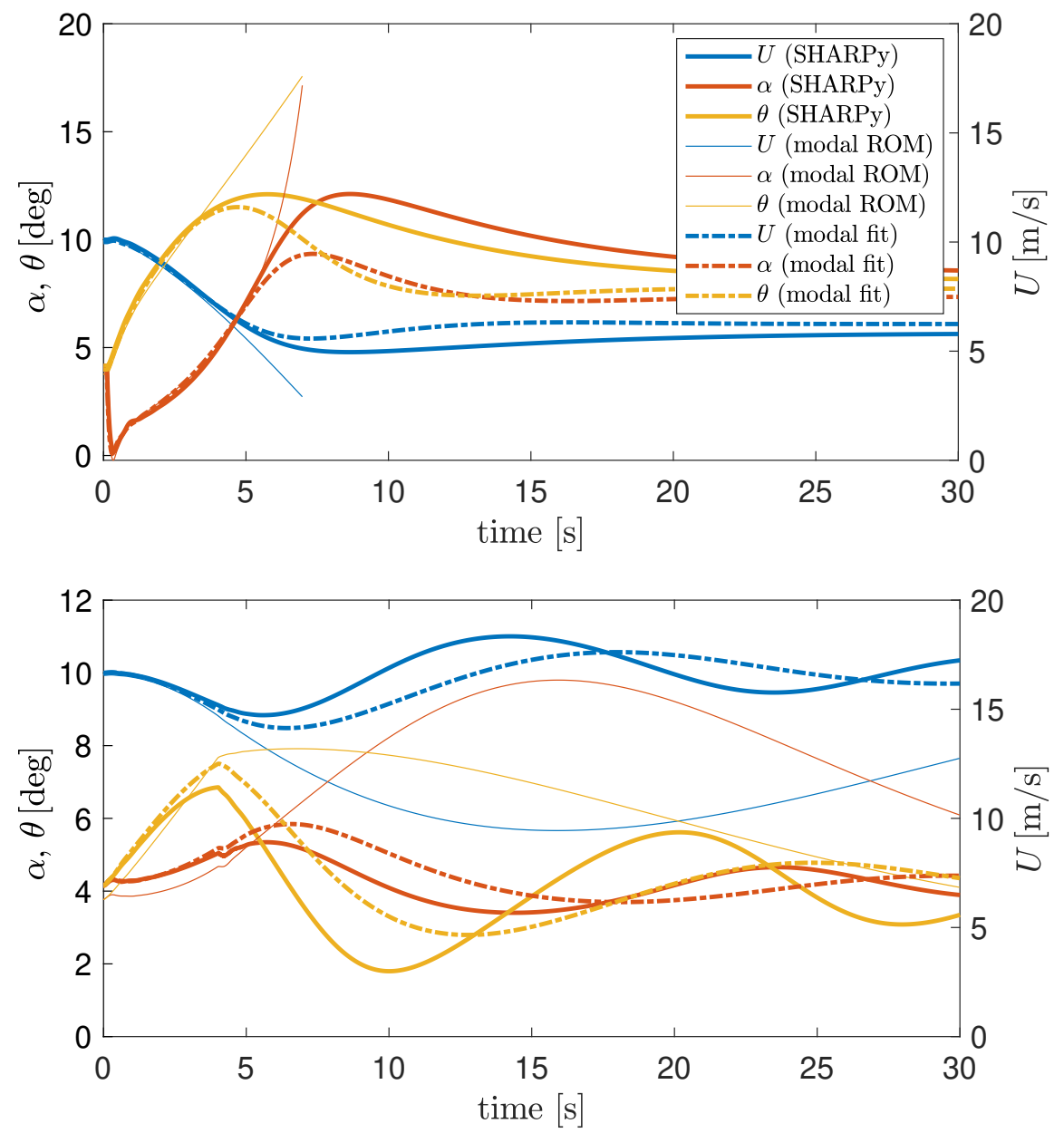

Fig. 2 Open loop response comparisons. Payload drop case (top) and elevator input case (bottom).

substantially improves the amplitudes of the predicted response, albeit showing a slight phase delay with respect to SHARPY simulations. It is believed that the main remaining source of discrepancy between the data-driven-enhanced modal-based internal models and the ground truth simulations is the truncation error due to the use of a limited number of modes (17 in this case, from which only 9 are active in these longitudinal cases), to which simulations of unconstrained structures are known to show increased sensitivity [19, ch. 6].

\section{Effect on Model Predictive Control (MPC) and Hoving Horizon Estimation (MHE) Performance}

In this subsection the effect of the data-driven improvements to our original internal model (8) on the closed-loop performance of an MPC controller is analysed. The MPC and MHE architectures used here are similar to those described in [20], but for completeness of notation we restate the relevant optimisation problems here. For MPC, we solve the following nonlinear optimal control problem over a time horizon $\tau_{c}$ at each sampling point $t_{i}=t_{i-1}+\tau_{s}$, where the 
(control) prediction horizon is split into $N_{c}$ intervals,

$$
\begin{array}{ll}
\min _{\boldsymbol{q}_{a e}^{k}, \boldsymbol{u}^{k}} & \frac{1}{2} \sum_{k=0}^{N_{c}-1}\left(\hat{\boldsymbol{q}}_{a e}^{k \top} Q_{c} \hat{\boldsymbol{q}}_{a e}^{k}+\hat{\boldsymbol{u}}^{k \top} R_{c} \hat{\boldsymbol{u}}^{k}\right)+\frac{1}{2} \hat{\boldsymbol{q}}_{a e}^{N_{c} \top} P_{c} \hat{\boldsymbol{q}}_{a e}^{N_{c}} \\
\text { s.t. } \quad & \boldsymbol{q}_{a e}^{0}=\boldsymbol{q}_{a e}^{c}, \\
& \boldsymbol{q}_{a e}^{k+1}=\boldsymbol{f}\left(\boldsymbol{q}_{a e}^{k}, \boldsymbol{u}^{k}, \boldsymbol{w}^{k}\right), \quad k=0, \cdots, N_{c}-1, \\
& \boldsymbol{u}_{l} \leq \boldsymbol{u}^{k} \leq \boldsymbol{u}_{u}, \quad k=0, \cdots, N_{c}-1, \\
& \boldsymbol{q}_{a e, l} \leq \boldsymbol{q}_{a e}^{k} \leq \boldsymbol{q}_{a e, u}, \quad k=1, \cdots, N_{c}, \\
& \frac{1}{2} \hat{\boldsymbol{q}}_{a e}^{N_{c} \top} P_{c} \hat{\boldsymbol{q}}_{a e}^{N_{c}} \leq a .
\end{array}
$$

where $\boldsymbol{q}_{a e}$ is a concatenated state containing all relevant states (e.g. structural, quarternion, aerodynamic, etc.) of the particular ROM considered, with $f$ is the corresponding discretized ODE model. The errors $\hat{\boldsymbol{q}}_{a e}=\boldsymbol{q}_{a e}-\overline{\boldsymbol{q}}_{a e}$ and $\hat{\boldsymbol{u}}=\boldsymbol{u}-\overline{\boldsymbol{u}}$ are defined with respect to a reference, equilibrium state $\overline{\boldsymbol{q}}_{a e}$ with control $\overline{\boldsymbol{u}}$ and disturbance $\overline{\boldsymbol{w}}$ (typically the trim solution, with $\overline{\boldsymbol{w}}=\mathbf{0}$ ). Matrices $Q_{c}, R_{c}$ and $P_{c}$ are positive definite state, input and final-state weights, $\boldsymbol{q}_{a e}^{c}$ is a prescribed initial condition, $\boldsymbol{u}_{l}, \boldsymbol{u}_{u}$ are control constraints, $\boldsymbol{q}_{a e, l}, \boldsymbol{q}_{a e, u}$ are state constraints and $a>0$ a final state constraint.

If full state information is known at every MPC sampling point $t_{i}$, the true state of the underlying system can be used for the initial condition $\boldsymbol{q}_{a e}^{0}$. In practice, however, such information is typically unavailable. Instead, an estimate of the true state can be provided by solving a Moving Horizon Estimation (MHE) problem. This takes a similar form to (15), in particular,

$$
\begin{array}{ll}
\min _{\boldsymbol{q}_{a e}^{e}, \boldsymbol{w}^{k}} & \frac{1}{2} \hat{\boldsymbol{q}}_{a e}^{e \top} P_{e} \hat{\boldsymbol{q}}_{a e}^{e}+\frac{1}{2} \hat{\boldsymbol{w}}^{-N_{e} \top} R_{e} \hat{\boldsymbol{w}}^{-N_{e}}+\sum_{k=-N_{e}}^{0} \frac{1}{2} \hat{\boldsymbol{y}}_{a e}^{k \top} Q_{e} \hat{\boldsymbol{y}}_{a e}^{k} \\
\text { s.t. } \quad & \boldsymbol{q}_{a e}^{-N_{e}}=\boldsymbol{q}_{a e}^{e}, \\
& \boldsymbol{q}_{a e}^{k+1}=\boldsymbol{f}\left(\boldsymbol{q}_{a e}^{k}, \boldsymbol{u}^{k}, \boldsymbol{w}^{k}\right), \quad k=-N_{e}, \cdots,-1, \\
& \boldsymbol{w}_{l} \leq \boldsymbol{w}^{k} \leq \boldsymbol{w}_{u}, \quad k=-N_{e}, \cdots,-1, \\
& \boldsymbol{q}_{a e, l} \leq \boldsymbol{q}_{a e}^{k} \leq \boldsymbol{q}_{a e, u}, \quad k=-N_{e}+1, \cdots, 0 .
\end{array}
$$

Here, the optimisation variables are the external disturbance $\boldsymbol{w}^{k}$ together with the initial state of the system $\boldsymbol{q}_{a e}^{e}$ at the beginning of the estimation horizon $\tau_{e}$ (i.e., the initial condition). The functional $f$ in $(16 \mathrm{c})$ is the same system as in [15), and terminal constraints on some of the states might be also considered (e.g., control surface deflections, part of the state space, are known at the sampling time). The cost function (16a) aims to penalise the error $\hat{\boldsymbol{y}}_{\boldsymbol{a} \boldsymbol{e}}=\boldsymbol{y}-\boldsymbol{y}_{\boldsymbol{e}}$ between a measured output $\boldsymbol{y}$ from the actual system and the estimated output produced by the internal model, which is chosen to be a linear function of the vector state, $\boldsymbol{y}_{\boldsymbol{e}}=\mathcal{L}\left(\boldsymbol{q}_{a e}\right)$. Penalties are applied over a horizon of length $N_{e}$. A quadratic cost function is built with a weighting matrix $Q_{e} \geqslant 0$. The cost function is also enhanced by the two first terms in (16a), where the errors $\hat{\boldsymbol{q}}_{a e}^{e}=\boldsymbol{q}_{a e}^{e}-\boldsymbol{q}_{a e, r}^{e}$ and $\hat{\boldsymbol{w}}^{-N_{e}}=\boldsymbol{w}^{-N_{e}}-\boldsymbol{w}_{r}$ are penalised, with $P_{e} \geqslant 0$ and $R_{e} \geqslant 0$. The quantities $\boldsymbol{q}_{a e, r}$ and $\boldsymbol{w}_{r}$ are reference state and disturbance vectors which are initialised with the reference values $\overline{\boldsymbol{q}}_{a e}$ and $\overline{\boldsymbol{w}}$ but are updated at every sampling point by the state and disturbances at the start of the second sub-interval of the previous MHE solution. This is done to improve initial convergence of the estimation problem, since it provides extra information of the reference configuration around which the estimation begins, and also of the subsequent ones, since the information of the previous estimations can be exploited.

Now that the MPC and MHE architectures have been described, we can now discuss the test-cases used to assess the performance of our data-driven ROMs in closed-loop. Two different problem setups are considered. The first is a disturbance rejection problem under gust perturbations, where different gust spans are considered, all with a considerable intensity of $20 \%$ the horizontal flight velocity. The second is the payload drop case from the previous example, where increasing values of the dropped payload are considered to determine the robustness of the controller in providing new stable configurations after the sudden change of the inertia distribution of the aircraft.

The external disturbance which must be estimated is a vector of external modal forces $\boldsymbol{\eta}$, added to the equation of motion in either (8) (linearised aero) or (12) (data-driven ROM), which gathers the effect of either the payload drop or the gust encounter on the system dynamics. A difference in the MPC/MHE implementation between the two cases is 
that in the gust-rejection setup, the disturbance estimated by the MHE is extrapolated in the MPC horizon by a linear decay over the first $N_{c} / 2$ intervals and then is set to zero over the remaining intervals, while in the payload drop case the disturbance is held constant throughout the entire MPC window. The internal models are constructed using the first 17 modes of vibration, including the 6 rigid-body modes, and a sampling time of $\tau_{s}=0.2 \mathrm{~s}$ is chosen. The estimator employs a horizon with $N_{e}=6(1.2 \mathrm{~s}, 0.59$ times the period of the first — bending — elastic mode) and the local translation velocities at five different locations along the main wing are the measurements $y$ fed to the MHE. The matrix $Q_{e}$ is taken equal to the identity, penalising errors in estimation equally for each of the sensors, and $P_{e}=I R_{e}=1$. A small stage penalty on the disturbance at each interval is also introduced, with weighing matrix $R=10^{-4}$. A constraint on the unit-norm of the quaternion associated to the frame $A$ has been added to the initial state of the first interval of the MHE window (the quaternion states at subsequent intervals automatically satisfy this constraint due to the dynamics (16c) constraint), so that it provides physically sensible rotation estimates. For the MPC, $N_{c}=12$ intervals are used $(2.4 \mathrm{~s})$. Control action is slightly penalised with $R_{c}=0.001 I$ and setting to 0.001 the entry of $Q_{c}$ corresponding to the aileron deflection (control surface deflections are part of the augmented state). The stage and terminal costs are chosen to penalise deviations in the trim velocity of the body attached frame $A$ by setting $P_{c}=Q_{c}=\operatorname{blkdiag}\left(\Phi\left(s_{A}\right)^{\top} \Phi\left(s_{A}\right), 0\right)$. Thrust input is constrained to be in a range of $[0,15] \mathrm{N}$ while elevator deflection and deflection rates are constrained to lie in the range $[-7.82,5.43] \mathrm{deg}$ and $\pm 7.48 \mathrm{deg} / \mathrm{s}$, to keep the control inputs within the upper and lower limits of the training data ensembles.

The two tested internal models differ only in that one is built using the data-driven model for the unsteady aerodynamics, as described in the section above, while the other one employs a reduced-order model $\left(A_{\Gamma}, B_{\Gamma}, C_{\Gamma}, D_{\Gamma}\right)$ of the unscaled linearised UVLM at a rigid-body angle of attack of 4 deg, obtained using the Krylov-based method of SHARPY. The latter results in a stable realisation with 68 aerodynamic states. Both employ the data-driven model for the gravitational forcing. The reference trim solution of both internal models have been forced to be for the same angle of attack, thrust setting and aileron deflection as the nonlinear solution provided by SHARPY to aid comparison. This requires applying a corrective clamping force to ensure that the vehicle maintains the required horizontal flight velocity, which gives an idea of the mismatch between the internal models and the nonlinear SHARPY trim solution, and also leads to different trim modal solutions. These corrective forces, normalised by the total weight of the aircraft $(W=78.25 \mathrm{~g} \mathrm{~N}$, including the entire payload), have been found to be $\left(\boldsymbol{f}_{A}^{\top}, \boldsymbol{m}_{A}^{\top}\right) / W=(-0.001,0,-0.066,0,-0.004 \mathrm{~m}, 0)$ and $\left(\boldsymbol{f}_{A}^{\top}, \boldsymbol{m}_{A}^{\top}\right) / W=(0.011,0,-0.214,0,0.094 \mathrm{~m}, 0)$ for the internal model using the data-driven aerodynamic module and the one based on the Krylov ROM, respectively, which already gives us an insight of the larger error of the latter model. As our controlled system we employ again the nonlinear aeroelasticity simulation host SHARPY, which communicates with our control framework using the UDP network described in [21] .

\section{Disturbance Rejection in Gust Encounters}
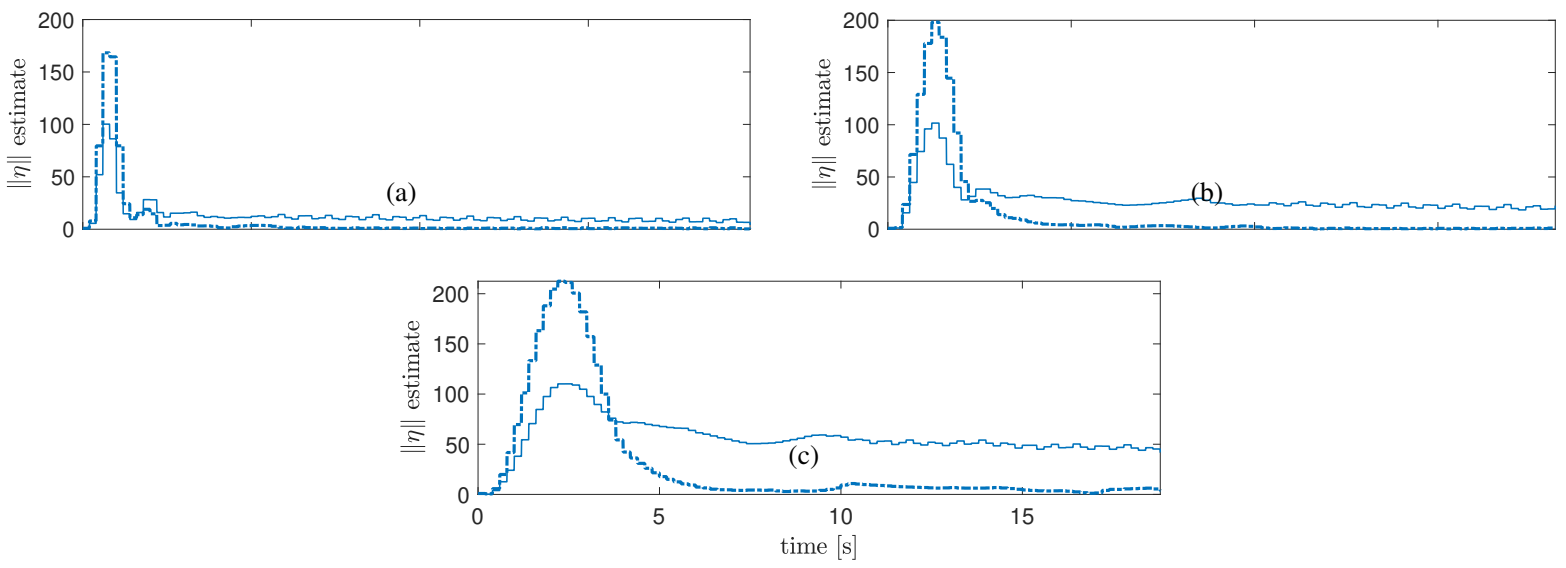

Fig. 3 Estimates of the disturbance magnitude for gust perturbations of span (a) $10 \mathrm{~m}$ (b) $20 \mathrm{~m}$ and (c) $40 \mathrm{~m}$. MPC closed-loop responses with the data-driven (-...) and linear ROM (_) UVLM models.

Here, three different gust profiles are considered as perturbations to the system and are simulated in SHARPY. All are 

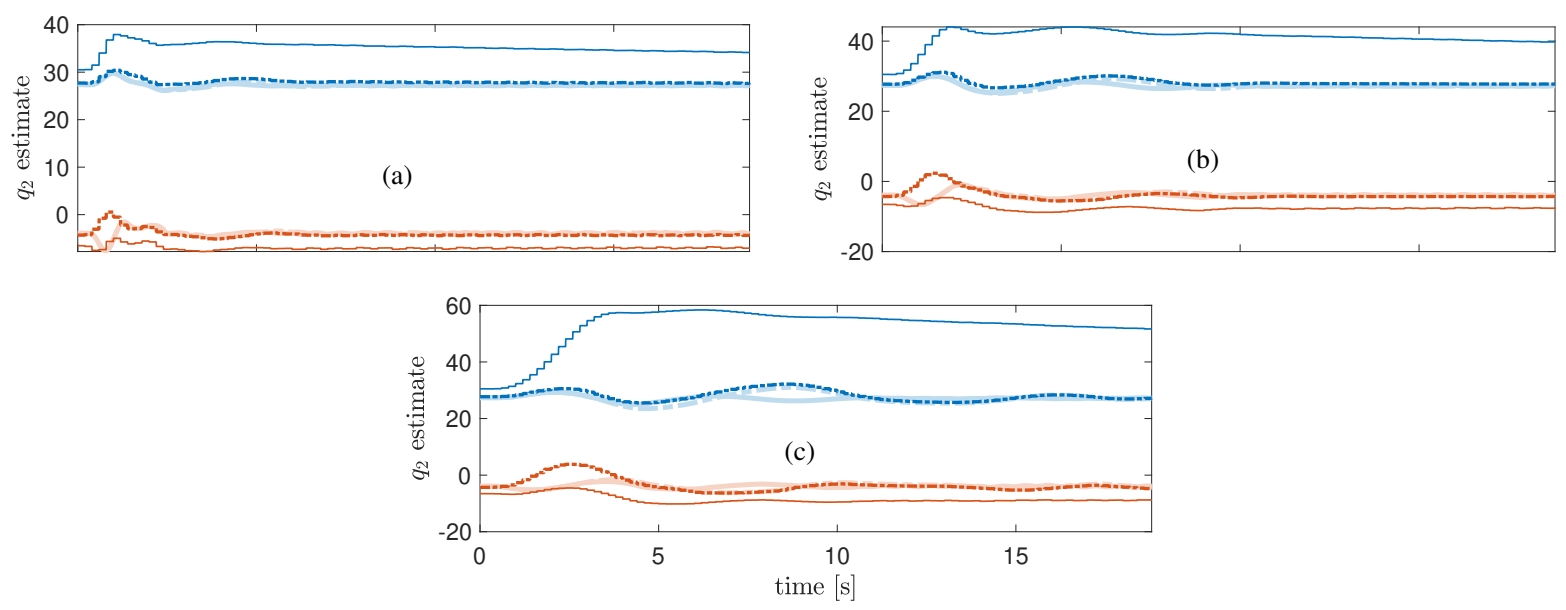

Fig. 4 Estimates of the $q_{2}$ state components (first bending in colour $\square$, second bending in $\square$ ) for gust perturbations of span (a) $10 \mathrm{~m}$ (b) $20 \mathrm{~m}$ and (c) $40 \mathrm{~m}$ for the data-driven (-...-) and linear ROM (-) UVLM internal models. Actual components from SHARPY closed-loop MPC simulations corresponding to the data-driven ( $\square \square$ ) and linear ROM ( $\square$ ) UVLM internal models.

1 - cos shaped gusts, defined by

$$
w_{g}(t)=\frac{W}{2}\left(1-\cos \left(\frac{\pi U_{\infty}\left(t-t_{g}\right)}{H_{g}}\right)\right)\left(\mathcal{H}\left(t_{g}\right)-\mathcal{H}\left(t_{g}+\frac{2 H_{g}}{U_{\infty}}\right)\right),
$$

with a vertical velocity amplitude of $W=2 \mathrm{~m} / \mathrm{s}$ (that is, an intensity of $20 \%$ ), reaching the leading edge of the aircraft at time $t_{g}=0.25 \mathrm{~s}$ and with increasing spans $H_{g}=\{10,20,40\} \mathrm{m}$. Here $\mathcal{H}(t)$ is the Heaviside step function at time $t$.

An outline of the presented results, gathered in four different figures, is given next, prior to its discussion. First, we show in Fig. 3 and Fig. 4 the results of the MHE: the first gathers the magnitude (norm) of the estimated external forcing vector (which has 9 components in total, for each longitudinal mode), $\boldsymbol{\eta}$, due to the gust perturbations. The second shows the two first components of the force/moment state $\boldsymbol{q}_{2}$ (which has 6 components in total, for each elastic longitudinal mode). These results should give us an idea of the effect of model-accuracy on the estimation problems (note that estimation of the velocity state $\boldsymbol{q}_{1}$ is rather uninteresting, since it is easily recovered by the estimator as all the considered measurements are velocities). The results obtained using the internal model employing data-driven aerodynamics are represented with dash-dotted lines (.....), and the one using the UVLM ROM, with thin solid line (-). Since we can easily obtain the components of $\boldsymbol{q}_{2}$ from post-processing of the closed-loop simulations in SHARPY, we include them in thick shaded lines in Fig. 4 for reference, similarly with dash-dotted $(\square \square \square)$ for those corresponding to the MPC closed-loop using the data-driven UVLM model of the form (12) and solid $(\square)$ for those corresponding to the MPC closed-loop using the linear ROM UVLM model of the form (8). The third figure, Fig. 5, shows the flight mechanics variables (flight velocity, $U$, angle of attack, $\alpha$, and pitch, $\theta$ ) of the open-loop responses, shown with thick solid lines ( - ), for the different gust perturbations together with the MPC closed-loop responses using the previously described line style notation, which has been employed consistently throughout all the figures in this section. Finally, the generated control inputs of both controllers are shown in Fig. 6, where the displayed signals are the normalised increments of control commands from the trim values.

We start the discussion inspecting the outcomes of the estimator. A first observation is that the controller equipped with the data-driven aerodynamic model is seen to produce much more accurate predictions of the system. As we observe in Fig. 3 the magnitude of the disturbances seems to be correctly captured by the data-driven-aero controller across all gust spans, showing a bump which eventually tends to zero once the aircraft transitions through the gust. Note that the external force caused by a $1-\cos$ gust does not follow exactly the same temporal distribution as the gust itself, due to the aerodynamic unsteadiness. Conversely, we observe that the ROM-based internal model has some difficulty estimating the external forcing, displaying a residual offset once the gust is no longer present. The magnitude of this error seems to correlate with the length of the gust. This might be explained by the larger excursions of the state space away from the region of validity of the UVLM ROM for more prolonged times. The results in Fig. 4 4 seem to corroborate the increase in accuracy of the data-driven aerodynamic model, which consistently finds estimates of the $\boldsymbol{q}_{2}$ state 

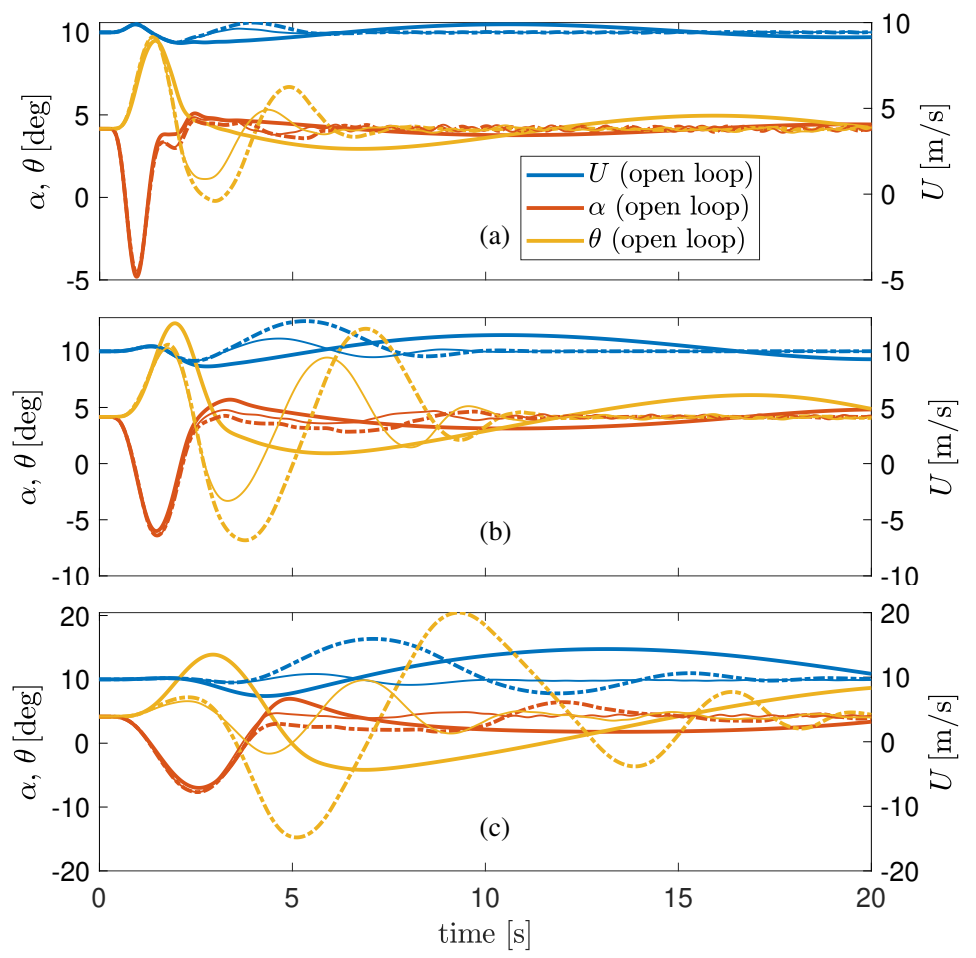

Fig. $5 U, \alpha$ and $\theta$ for gust perturbations of span (a) $10 \mathrm{~m}$ (b) $20 \mathrm{~m}$ and (c) $40 \mathrm{~m}$, with open loop ( MPC closed-loop responses using the data-driven (.....) and the linear ROM (- ${ }^{-}$UVLM models.

with very good agreement with the post-processed closed-loop solution of SHARPY. The first two components, which correspond to the physically more significant first and second bending modes of the aircraft, are seen to match almost perfectly the values retrieved from SHARPY, with the second (red lines) deviating slightly during the main excitation however rapidly coalescing with the reference. On the contrary, the internal model employing the linearised UVLM ROM (8) even initially exhibits discrepancies from the trim values and produces estimates with errors that grow both with time and with increasing gust spans.

We now focus on control performance. By observing the responses of the flight variables in Fig. 5. we see that both controllers are capable of stabilising the system consistently across the different explored gust spans. We note that since the gusts are all of the same intensity, greater spans $H_{g}$ translate into more prolonged excitations to the system, which is observed by larger-amplitude responses of the open-loop dynamics. Another observation is that the internal model employing the linearised UVLM ROM appears to produce more effective stabilisation when compared to the one equipped with the data-driven model, dampening the system faster (i.e., with shorter settling time) and producing a less oscillatory response. This is further supported by the control inputs shown in Fig. 6, where the control effort of the UVLM-ROM-based internal controller appears to be consistently lower for all the tested cases. As a matter of fact, the ROM-based control effort is roughly half that of the data-driven-aero for the first two cases, and a sixth for the third case (where the control effort is computed by adding up the weighted norm $\hat{\boldsymbol{u}}^{\top} R_{c} \hat{\boldsymbol{u}}+10^{-3} \hat{\boldsymbol{\delta}}_{e}{ }^{2}$ over all time steps of the control input signals displayed in Fig. 6); the data-driven based internal model produces inputs with longer settling times but in general smoother than the ROM-based counterpart, especially in the thrust setting input (top-right column in Fig. 6.

An explanation of these apparent contradictory results may lie in the fact that the posed MPC problem is rather benign, since the control objective is to penalise deviations in the velocity of the frame $A$ while using velocity measurements for the estimation. Therefore, even with poorly estimated values of the disturbances (which might compensate for inaccuracies in the predicted aerodynamic forces), the ROM-based internal model accurately estimates the velocity states $\boldsymbol{q}_{1}$ and the controller is able to produce more efficient control laws. Note that inputs on control surfaces are very well approximated by the linear UVLM (deflections are usually kept within the linear regime), and it is large variations in elastic deformation, orientation or rigid-body velocities which are known important sources of error in the linearised 

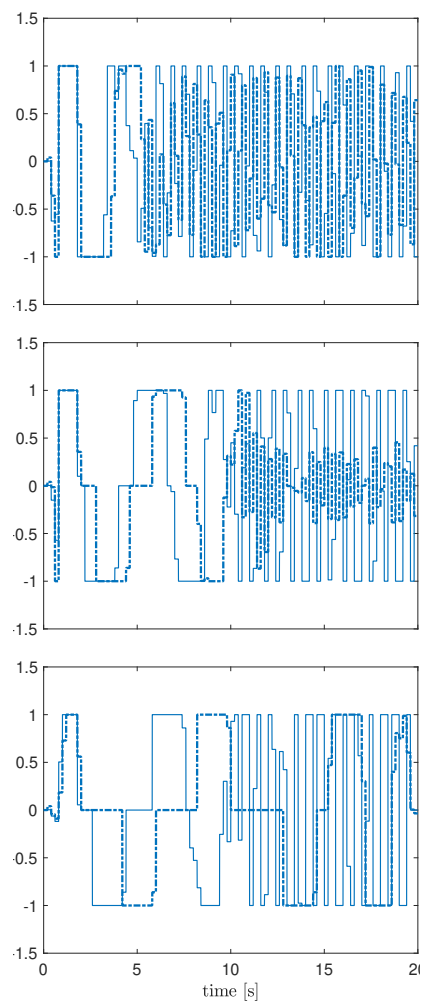
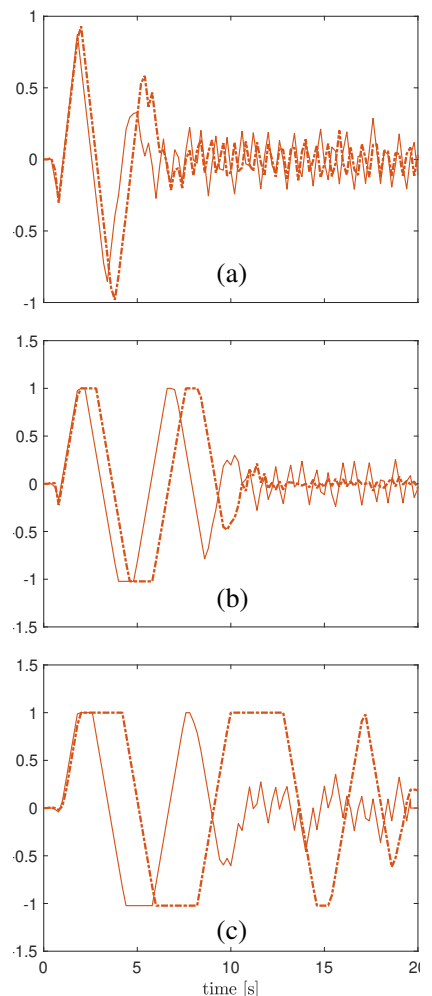
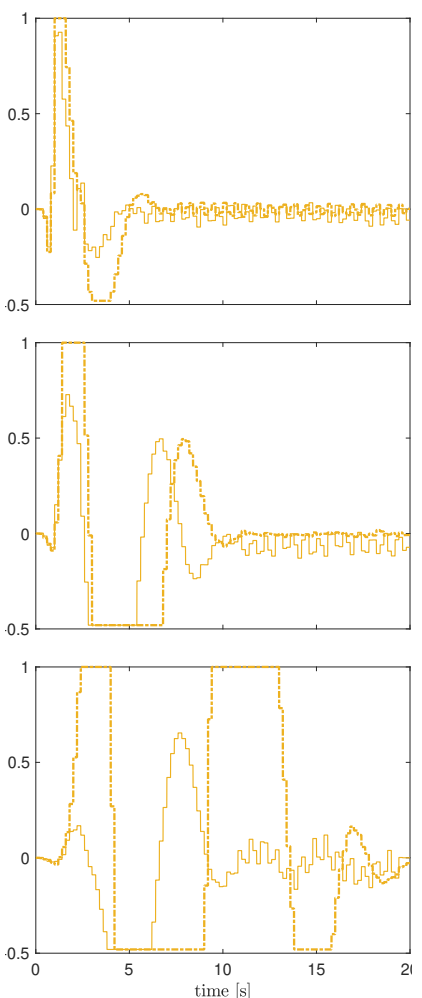

Fig. 6 Normalised control inputs: elevator rate $\dot{\delta}(-)$, deflection $\delta(-)$ and thrust $T(-)$ for gust perturbations of span (a) $10 \mathrm{~m}$ (b) $20 \mathrm{~m}$ and (c) $40 \mathrm{~m}$. MPC closed-loop responses with the data-driven (......) and linear ROM (-) UVLM models.

UVLM. Therefore, the linear-aerodynamics-based controller could benefit from a better prediction of the effect of elevator inputs on the aerodynamic forces, while the data-driven input-output mappings might see a deterioration along the closed-loop solution (possibly due to unexplored combination of inputs in the data-acquisition simulations).

\section{Payload Drop Stabilisation}

In this case, the nonlinear aeroelastic simulations of the HALE vehicle in SHARPY are set up so that at $t=0.25 \mathrm{~s} \mathrm{a}$ fraction of the aircraft payload is released, which introduces a sudden perturbation to the trim flight conditions. Three different magnitudes of the dropped payload are considered, $m_{l}=-25,-35,-45 \mathrm{~kg}$, with the first one giving the same problem setup that has been explored in the example of Section II.D

The magnitudes that we show in this case are the same as those presented in the previous gust-rejection scenario, with the estimated values of the external forcing magnitude and the first two components of the modal force/moment state $\boldsymbol{q}_{2}$ presented in Fig. 7 and Fig. 88, the response in flight mechanics variables (flight velocity, $U$, angle of attack, $\alpha$, and pitch, $\theta$ ) shown in Fig. 9 and the generated control inputs of both controllers gathered in Fig. 10 The employed line style in the figures below is consistent with the one used in the previous gust-rejection setting.

The superior model accuracy provided by the data-driven aerodynamic model is also observed here in both the external force $\boldsymbol{\eta}$ and the modal force state $\boldsymbol{q}_{2}$ estimation. As seen in Fig. 7 , the magnitude of the estimated external force by the data-driven aerodynamic model is seen to reach a roughly constant value after a reasonable settling time, which is in agreement with the nature of the disturbance (note that after stabilisation, the dropped mass would add a constant forcing input to the equations of motion). Moreover, the magnitude seems to scale correspondingly with the increase in the mass being released. Conversely, the estimated values by the linear-aerodynamics-based internal model are seen, in the same figure, to either diverge or oscillate. The slight divergence, observable with both internal models, is due to a drift in the the projection onto the first bending mode of the external forcing. This deviation is related to the corresponding first bending mode component of $\boldsymbol{q}_{2}$, shown in blue in Fig. 8, which is also seen to drift with time in the estimates provided by both internal models. This can be caused by the MHE reaching an alternative 

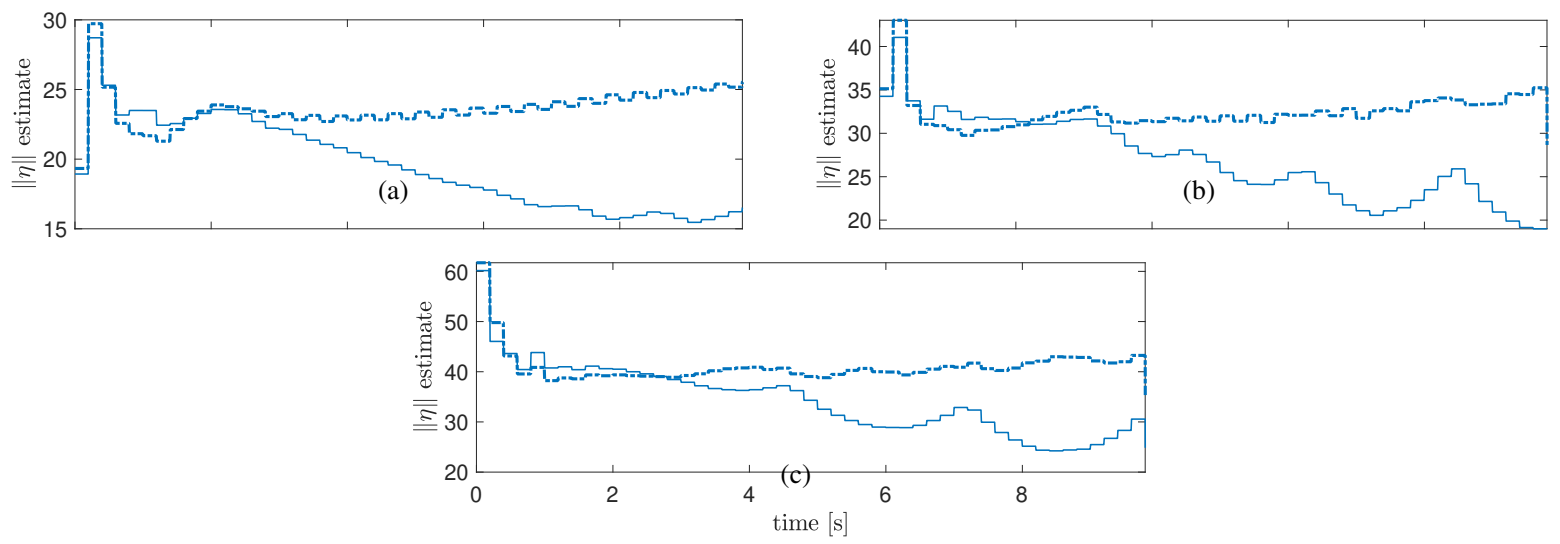

Fig. 7 Estimates of the disturbance magnitude for the payload mass releases of (a) $25 \mathrm{~kg}$ (b) $35 \mathrm{~kg}$ and (c) $45 \mathrm{~kg}$. MPC closed-loop responses with the data-driven (.....) and linear ROM (- ${ }_{-}$UVLM models.
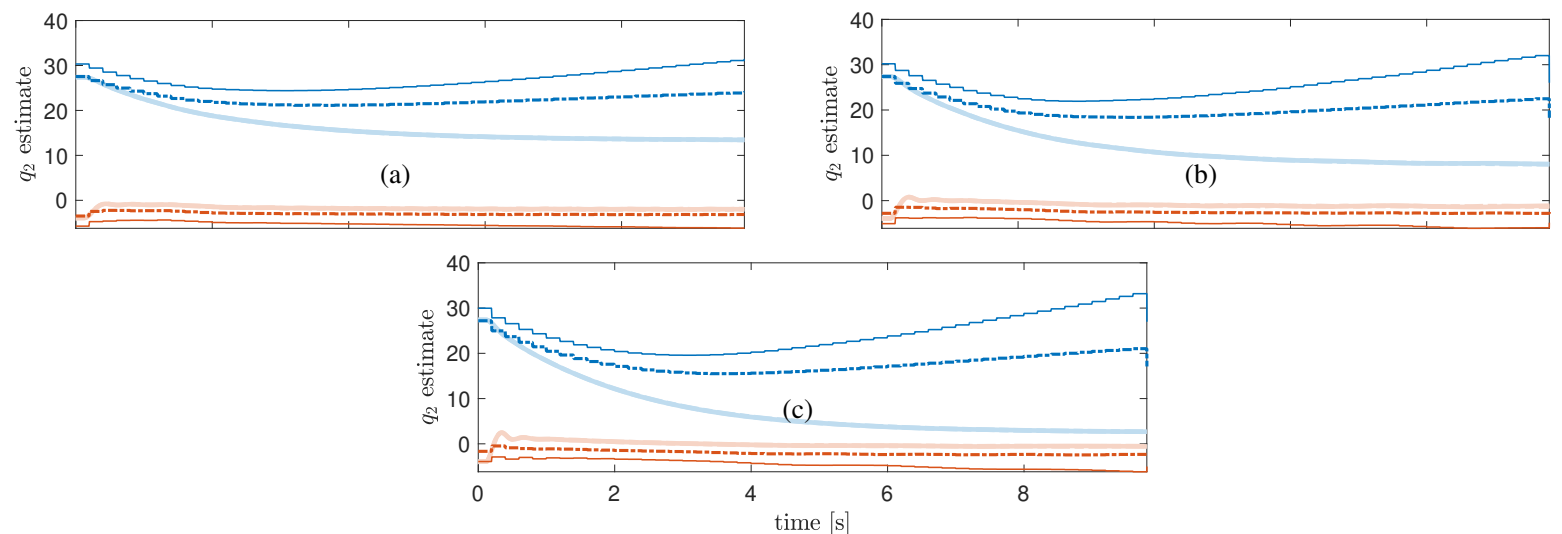

Fig. 8 Estimates of the $q_{2}$ state components (first bending in colour $\square$, second bending in $\square$ ) for the payload mass releases of (a) $25 \mathrm{~kg}$ (b) $35 \mathrm{~kg}$ and (c) $45 \mathrm{~kg}$ for the data-driven (.....) and linear ROM (internal models. Actual components from SHARPY closed-loop MPC simulations corresponding to the datadriven ( $\square-\square)$ and linear ROM ( $\square$ ) UVLM internal models.

local minimum which compensates bending deformation with external forcing in the bending component and/or by the non-negligible model mismatch between both models and SHARPY nonlinear simulations. To overcome this difficulty, additional measurements (e.g., root moments or strains) could be considered. Nevertheless, the remaining estimated components of $\boldsymbol{q}_{2}$ produced by the data-driven-aero model (Fig. 8 only shows the two first modes, for clarity) are very good and enable successful stabilisation of the system.

The flight variables in Fig. 9 show us the challenge of the posed control case, where the open-loop simulations see the angle of attack and pitch increase rapidly, which could possibly lead to stall in a realistic scenario. As a matter of fact, the two last cases yield angles of attack higher than $20 \mathrm{deg}$ for which the validity of the UVLM is compromised (note that no stall effects are modelled). A first compelling observation is that both tested internal models achieve very effective stabilisation of the aircraft. And in contrast to the gust-rejection scenario portrayed above, the linear-aerodynamics-based controller shows worse performance than the data-driven-aero counterpart, illustrated by growing oscillations in the flight variables (principally pitch) of the two heaviest release cases. This deterioration on the performance is seen with greater ease in the resulting input commands shown in Fig. 10, which show divergent oscillations in both the elevator and thrust setting inputs. The control effort of the signals displayed in Fig.10 (computed as before) are roughly the same for both internal models for the two first payload releases (where the cost of the larger-amplitude elevator and thrust oscillations of the ROM-based controller are balanced by the higher oscillatory response of the elevator rate for the data-driven-aero model), however for the heaviest release case the ROM-based internal model requiring $33.5 \%$ more effort than the data-driven counterpart. 

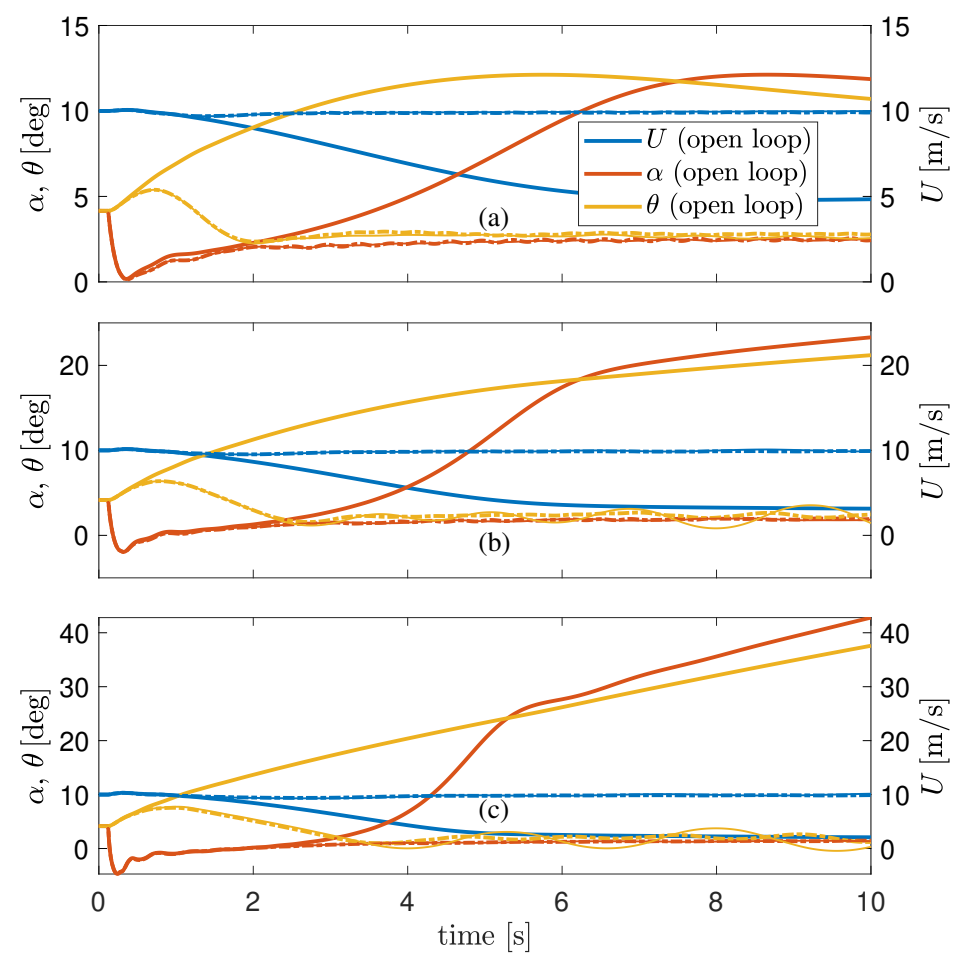

Fig. $9 U, \alpha$ and $\theta$ for the payload mass releases of (a) $25 \mathrm{~kg}$ (b) $35 \mathrm{~kg}$ and (c) $45 \mathrm{~kg}$ with open loop (—) and MPC closed-loop responses using the data-driven (.....) and the linear ROM (-

Therefore, it is suggested that, in this more complex control case, where the system has to be stabilised around a notably different deformed configuration (precisely where the linearised UVLM is known to produce less accurate predictions) the internal model based on data-driven aerodynamics can exploit the improved accuracy of the model to better stabilise the system against the considered range of disturbances showing better performance than the ROM-based counterpart.

\section{Conclusions}

We have investigated computationally-efficient strategies to incorporate critical missing nonlinear physics in an internal model for control. Data-driven regression tools have proved successful to obtain closed models of the gravity forcing and the unsteady aerodynamics in terms of the modal intrinsic variables. Regarding the gravity terms, data-driven identification of the forcing field avoids the necessity to introduce approximations to the entire quaternion field. Instead, only the quaternion at one single spatial coordinate needs to be tracked. This yields a reduction of the the state-space vector of the order of the number of retained modes. The unsteady aerodynamics are fundamentally more complex. When stable identified models of the unsteady aerodynamic models have been obtained, these have been shown to be more accurate, accounting for varying dynamic pressure and induced drag. Clear improvements with respect linear aerodynamic models have been shown in comparisons of the long-time open-loop simulations against full nonlinear solutions. However, when the data-driven enhanced models are used within the MPC/MHE framework their effect on the close- loop performance has been found so far inconclusive. On the one hand, it has been observed that the increased accuracy of the internal models yields better estimation performance, however, on the other hand, no significant improvements in control performance could be observed. A nonlinear control problem where these improvements are highlighted further is yet a matter of future investigation.

\section{References}

[1] Afonso, F., Vale, J., Oliveira, E., Lau, F., and Suleman, A., "A review on non-linear aeroelasticity of high aspect-ratio wings," Progress in Aerospace Sciences, Vol. 89, 2017, pp. 40-57. https://doi.org/https://doi.org/10.1016/j.paerosci.2016.12.004 

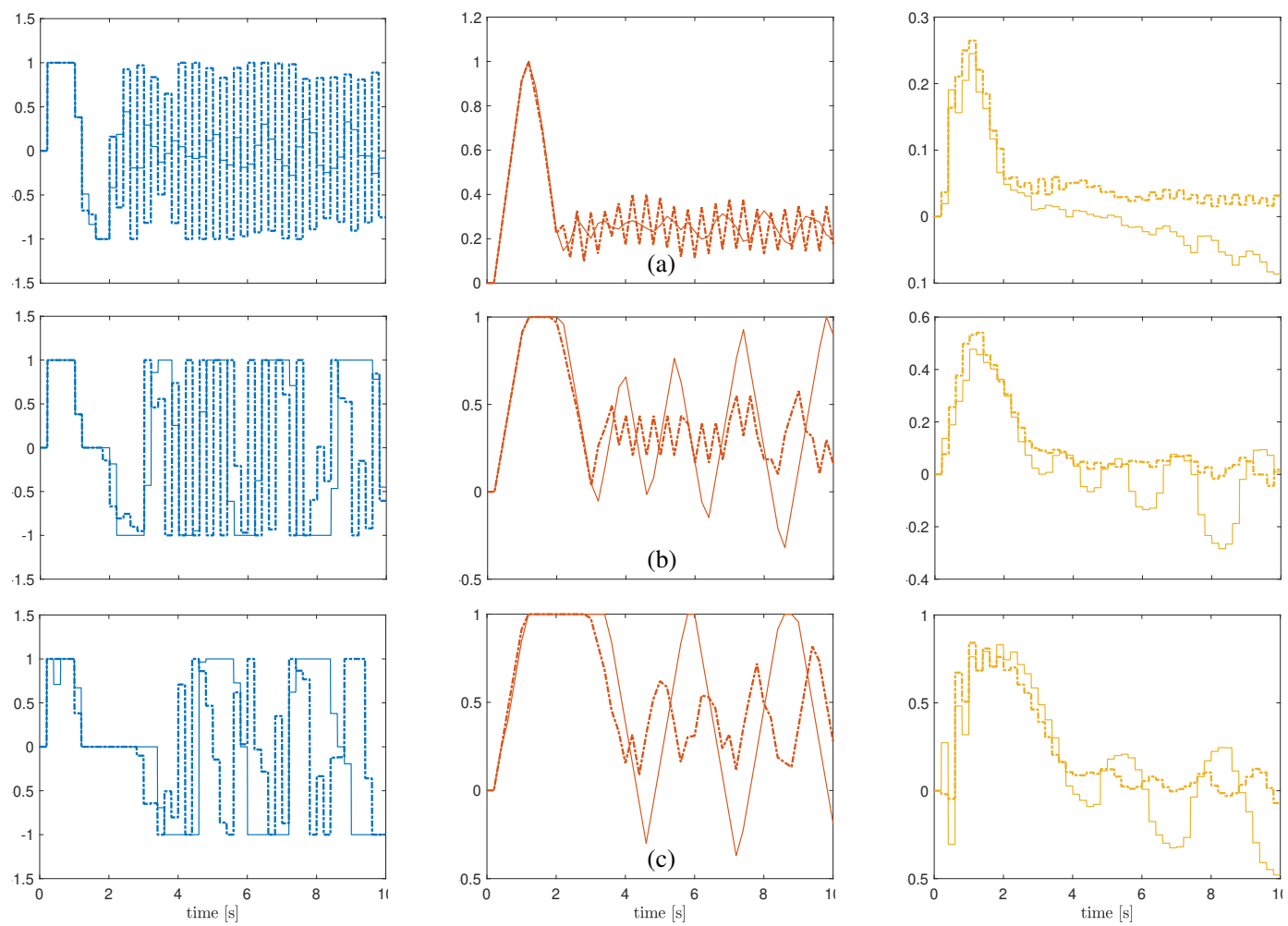

Fig. 10 Normalised control inputs: elevator rate $\dot{\delta}(-)$, deflection $\delta(-)$ and thrust $T$ ( $\longrightarrow$ ) for the payload mass releases of (a) $25 \mathrm{~kg}$ (b) $35 \mathrm{~kg}$ and (c) $45 \mathrm{~kg}$. MPC closed-loop responses with the data-driven (....-.) and linear ROM (—) UVLM models.

[2] Kopf, M., Bullinger, E., Giesseler, H.-g., Adden, S., and Findeisen, R., "Model Predictive Control for Aircraft Load Alleviation: Opportunities and Challenges," 2018 American Control Conference (ACC), 2018, pp. 2417-2424. https: //doi.org/10.23919/ACC.2018.8430956.

[3] Wang, Y., Wynn, A., and Palacios, R., "Nonlinear Aeroelastic Control of Very Flexible Aircraft Using Model Updating," Journal of Aircraft, Vol. 55, No. 4, 2018, pp. 1551-1563. https://doi.org/https://doi.org/10.2514/1.C034684

[4] Pereira, M., Kolmanovsky, I., Cesnik, C. E., and Vetrano, F., "Model Predictive Control Architectures for Maneuver Load Alleviation in Very Flexible Aircraft," AIAA Scitech 2019 Forum, San Diego, CA, 2019. https://doi.org/https: //doi.org/10.2514/6.2019-1591

[5] Diehl, M., Bock, H., and Schlöder, J., "A Real-Time Iteration Scheme for Nonlinear Optimization in Optimal Feedback Control," SIAM Journal on Control and Optimization, Vol. 43, No. 5, 2005, pp. 1714-1736. https://doi.org/https://doi.org/10.1137/ S0363012902400713

[6] Barzgaran, B., Quenzer, J. D., Mesbahi, M., Morgansen, K. A., and Livne, E., "Real-time Model Predictive Control for Gust Load Alleviation on an Aeroelastic Wind Tunnel Test Article," AIAA Scitech 2021 Forum, Reston, Virginia, 2021. https://doi.org/10.2514/6.2021-0500

[7] Artola, M., Wynn, A., and Palacios, R., "Modal-Based Nonlinear Model Predictive Control for 3D Very Flexible Structures," IEEE Transactions on Automatic Control, 2021, pp. 1-1. https://doi.org/10.1109/TAC.2021.3071326

[8] Artola, M., Goizueta, N., Wynn, A., and Palacios, R., "Proof of Concept for a Hardware-in-the-Loop Nonlinear Control Framework for Very Flexible Aircraft," AIAA Scitech Forum, Nashville, Tennessee, USA, 2021. https://doi.org/10.2514/6.20211392.

[9] Maraniello, S., and Palacios, R., "Parametric Reduced-Order Modeling of the Unsteady Vortex-Lattice Method," AIAA Journal, Vol. 58, No. 5, 2020, pp. 2206-2220. https://doi.org/10.2514/1.j058894 
[10] Goizueta, N., Wynn, A., and Palacios, R., "Parametric krylov-based order reduction of aircraft aeroelastic models," AIAA Scitech 2021 Forum, 2021.

[11] Hodges, D. H., "Geometrically Exact, Intrinsic Theory for Dynamics of Curved and Twisted Anisotropic Beams," AIAA Journal, 2003. https://doi.org/https://doi.org/10.2514/2.2054

[12] Palacios, R., "Nonlinear Normal Modes in an Intrinsic Theory of Anisotropic Beams," Journal of Sound and Vibration, Vol. 330, No. 8, 2011, pp. 1772-1792. https://doi.org/https://doi.org/10.1016/j.jsv.2010.10.023.

[13] Murua, J., Palacios, R., and Graham, J. M. R., "Applications of the Unsteady Vortex-Lattice Method in Aircraft Aeroelasticity and Flight Dynamics," Progress in Aerospace Sciences, Vol. 55, 2012, pp. 46 - 72. https://doi.org/https://doi.org/10.1016/j. paerosci.2012.06.001

[14] Deskos, G., del Carre, A., and Palacios, R., "Assessment of Low-Altitude Atmospheric Turbulence Models for Aircraft Aeroelasticity,” Journal of Fluids and Structures, Vol. 95, 2020, p. 102981. https://doi.org/https://doi.org/10.1016/j.jfluidstructs. 2020.102981

[15] del Carre, A., Muñoz-Simón, A., Goizueta, N., and Palacios, R., "SHARPy: A Dynamic Aeroelastic Simulation Toolbox for Very Flexible Aircraft and Wind Turbines," Journal of Open Source Software, Vol. 4, No. 44, 2019, p. 1885. https://doi.org/https://doi.org/10.21105/joss.01885

[16] Geradin, M., and Cardona, A., Flexible Multibody Dynamics: a Finite Element Approach, $3^{\text {rd }}$ ed., John Wyley and Sons, Chichester, UK, 2015.

[17] Simo, J., and Vu-Quoc, L., "On the Dynamics in Space of Rods Undergoing Large Motions — A Geometrically Exact Approach," Computer Methods in Applied Mechanics and Engineering, Vol. 66, No. 2, 1988, pp. 125 - 161. https://doi.org/10.1016/00457825(88)90073-4, URL http://www.sciencedirect.com/science/article/pii/0045782588900734.

[18] Schlegel, M., and Noack, B. R., “On long-term boundedness of Galerkin models,” Journal of Fluid Mechanics, Vol. 765, 2015, p. 325-352. https://doi.org/10.1017/jfm.2014.736

[19] Wang, Y., “Aeroelastic Modelling and Control of Very Flexible Air Vehicles Using a Nonlinear Modal Formulation,” Ph.D. thesis, Imperial College London, 2015.

[20] Artola, M., Goizueta, N., Wynn, A., and Palacios, R., "Aeroelastic Control and Estimation with a Minimal Nonlinear Modal Description,” AIAA Journal, Vol. 59, No. 7, 2021, pp. 2697-2713.

[21] Artola, M., Goizueta, N., Wynn, A., and Palacios, R., "Proof of Concept for a Hardware-in-the-Loop Nonlinear Control Framework for Very Flexible Aircraft," AIAA Scitech 2021 Forum, Nashville, TN, 2021. https://doi.org/10.2514/6.2021-1392 